\title{
DESIGN OF RATIONAL ROTATION-MINIMIZING RIGID BODY MOTIONS BY HERMITE INTERPOLATION
}

\author{
RIDA T. FAROUKI, CARLOTTA GIANNELLI, CARLA MANNI, \\ AND ALESSANDRA SESTINI
}

\begin{abstract}
The construction of space curves with rational rotationminimizing frames (RRMF curves) by the interpolation of $G^{1}$ Hermite data, i.e., initial/final points $\mathbf{p}_{i}$ and $\mathbf{p}_{f}$ and frames $\left(\mathbf{t}_{i}, \mathbf{u}_{i}, \mathbf{v}_{i}\right)$ and $\left(\mathbf{t}_{f}, \mathbf{u}_{f}, \mathbf{v}_{f}\right)$, is addressed. Noting that the RRMF quintics form a proper subset of the spatial Pythagorean-hodograph (PH) quintics, characterized by a vector constraint on their quaternion coefficients, and that $C^{1}$ spatial PH quintic Hermite interpolants possess two free scalar parameters, sufficient degrees of freedom for satisfying the RRMF condition and interpolating the end points and frames can be obtained by relaxing the Hermite data from $C^{1}$ to $G^{1}$. It is shown that, after satisfaction of the RRMF condition, interpolation of the end frames can always be achieved by solving a quadratic equation with a positive discriminant. Three scalar freedoms then remain for interpolation of the end-point displacement $\mathbf{p}_{f}-\mathbf{p}_{i}$, and this can be reduced to computing the real roots of a degree 6 univariate polynomial. The nonlinear dependence of the polynomial coefficients on the prescribed data precludes simple a priori guarantees for the existence of solutions in all cases, although existence is demonstrated for the asymptotic case of densely-sampled data from a smooth curve. Modulation of the hodograph by a scalar polynomial is proposed as a means of introducing additional degrees of freedom, in cases where solutions to the end-point interpolation problem are not found. The methods proposed herein are expected to find important applications in exactly specifying rigid-body motions along curved paths, with minimized rotation, for animation, robotics, spatial path planning, and geometric sweeping operations.
\end{abstract}

\section{INTRODUCTION}

The distinctive feature of a polynomial Pythagorean-hodograph (PH) curve $\mathbf{r}(\xi)$ is that the parametric speed $\sigma(\xi)=\left|\mathbf{r}^{\prime}(\xi)\right|$ is a polynomial (rather than the square root of a polynomial) in the curve parameter $\xi$. Many significant computational advantages stem from this fact [5]. However, the nonlinear nature of the algebraic models used to characterize PH curves precludes easy construction by the standard "control polygon" paradigm of computer-aided geometric design [4]. Instead, the geometrical construction of PH curves is typically achieved by the solution of Hermite interpolation problems.

Algorithms for Hermite and spline interpolation by planar PH curves are generally based upon the complex representation [1, 13, 14, 18. Interpolation of $C^{1}$

Received by the editor December 24, 2009 and, in revised form, January 16, 2011.

2010 Mathematics Subject Classification. Primary 65-XX, 53-XX.

Key words and phrases. Pythagorean-hodograph curves, quaternions, angular velocity, Hermite interpolation, rigid body motion, rational rotation-minimizing frames.

(c)2011 American Mathematical Society Reverts to public domain 28 years from publication 
Hermite data (i.e., given initial/final points and derivatives $\mathbf{r}(0)=\mathbf{p}_{i}, \mathbf{r}(1)=\mathbf{p}_{f}$ and $\left.\mathbf{r}^{\prime}(0)=\mathbf{d}_{i}, \mathbf{r}^{\prime}(1)=\mathbf{d}_{f}\right)$ using the quaternion form of spatial PH quintics $\mathbf{r}(\xi)$ was first studied in [8]. It was observed that the solutions of this problem incur three free angular parameters $\phi_{0}, \phi_{1}, \phi_{2}$; but the interpolants depend only upon their differences, so one may choose $\phi_{1}=0$ (say), without loss of generality. Strategies for the "optimal" selection of the remaining two parameters $\phi_{0}, \phi_{2}$ were subsequently considered in 9 ; see also 24 .

The rational rotation-minimizing frame (RRMF) curves are a subset of the spatial PH curves, with the additional property that they admit rational orthonormal frames $(\mathbf{t}, \mathbf{u}, \mathbf{v})$ such that the normal-plane vectors $\mathbf{u}, \mathbf{v}$ exhibit no instantaneous rotation about the curve tangent $\mathbf{t}=\mathbf{r}^{\prime} /\left|\mathbf{r}^{\prime}\right|$, i.e., rotation-minimizing frames (RMFs). Such frames are extremely useful for animation, motion planning, robotics, swept surface constructions, and related problems [19, 21, 25]. The ability to compute exact RMFs with a rational dependence on the curve parameter eliminates the need for data-intensive and potentially error-prone approximation schemes; see [12, 20, 25, 26].

The variation of a frame $(\mathbf{t}, \mathbf{u}, \mathbf{v})$ defined on a curve $\mathbf{r}(\xi)$ is specified by its vector angular velocity $\boldsymbol{\omega}(\xi)$, through the relations

$$
\frac{\mathrm{d} \mathbf{t}}{\mathrm{d} s}=\boldsymbol{\omega} \times \mathbf{t}, \quad \frac{\mathrm{d} \mathbf{u}}{\mathrm{d} s}=\boldsymbol{\omega} \times \mathbf{u}, \quad \frac{\mathrm{d} \mathbf{v}}{\mathrm{d} s}=\boldsymbol{\omega} \times \mathbf{v},
$$

where $s$ is the arc length along $\mathbf{r}(\xi)$. The magnitude and direction of $\boldsymbol{\omega}$ specify the frame angular speed and rotation axis at each point. The characteristic property of an RMF is that its angular velocity has no component along $\mathbf{t}$, i.e., $\boldsymbol{\omega} \cdot \mathbf{t} \equiv 0$ (equivalently, the derivatives $\mathbf{u}^{\prime}$ and $\mathbf{v}^{\prime}$ are always parallel to $\mathbf{t}$ ). The familar Frenet frame $(\mathbf{t}, \mathbf{p}, \mathbf{b})$, where the principal normal $\mathbf{p}$ points to the center of curvature and $\mathbf{b}=\mathbf{t} \times \mathbf{p}$ is the binormal, is not rotation-minimizing since its angular velocity is defined 22 by the Darboux vector $\boldsymbol{\omega}=\kappa \mathbf{b}+\tau \mathbf{t}$, where $\kappa$ and $\tau$ are the curvature and torsion of $\mathbf{r}(\xi)$. A rotation-minimizing frame, on the other hand, has angular velocity $\boldsymbol{\omega}=\kappa \mathbf{b}$.

The intent of this paper is to develop algorithms for interpolation of $G^{1}$ Hermite "motion data" by RRMF curves 1 that will facilitate their practical use in diverse applications. Such data comprises initial/final points $\mathbf{p}_{i}$ and $\mathbf{p}_{f}$ and frames $\left(\mathbf{t}_{i}, \mathbf{u}_{i}, \mathbf{v}_{i}\right)$ and $\left(\mathbf{t}_{f}, \mathbf{u}_{f}, \mathbf{v}_{f}\right)$ that specify the initial/final positions and orientations for the smooth motion of a rigid body. The output is an RRMF curve $\mathbf{r}(\xi)$ and associated frame $(\mathbf{t}(\xi), \mathbf{u}(\xi), \mathbf{v}(\xi))$ defined on $\xi \in[0,1]$ where $\mathbf{t}(\xi)=\mathbf{r}^{\prime}(\xi) /\left|\mathbf{r}^{\prime}(\xi)\right|$ is the unit tangent to $\mathbf{r}(\xi)$, and

$$
\begin{array}{ll}
\mathbf{r}(0)=\mathbf{p}_{i}, & (\mathbf{t}(0), \mathbf{u}(0), \mathbf{v}(0))=\left(\mathbf{t}_{i}, \mathbf{u}_{i}, \mathbf{v}_{i}\right), \\
\mathbf{r}(1)=\mathbf{p}_{f}, & (\mathbf{t}(1), \mathbf{u}(1), \mathbf{v}(1))=\left(\mathbf{t}_{f}, \mathbf{u}_{f}, \mathbf{v}_{f}\right) .
\end{array}
$$

Furthermore, the orthonormal frame vectors $(\mathbf{t}(\xi), \mathbf{u}(\xi), \mathbf{v}(\xi))$ have a rational dependence on the curve parameter $\xi$, and the frame angular velocity $\boldsymbol{\omega}(\xi)$ maintains a vanishing component along $\mathbf{t}(\xi)$, i.e., $\mathbf{u}(\xi)$ and $\mathbf{v}(\xi)$ exhibit no instantaneous rotation about $\mathbf{t}(\xi)$. This defines the "most natural" variation for the orientation of a rigid body traversing the path $\mathbf{r}(\xi)$ in such a manner that one principal axis is always aligned with the tangent vector $\mathbf{t}(\xi)$.

\footnotetext{
${ }^{1}$ Preliminary results on interpolation of "ordinary" Hermite data (i.e., just end points $\mathbf{p}_{i}, \mathbf{p}_{f}$ and tangents $\mathbf{t}_{i}, \mathbf{t}_{f}$ ) using the Hopf map form of RRMF curves may be found in 11.
} 
It must be emphasized that the shape quality of solutions to this problem can be rather sensitive to the initial data. For example, in cases where the vectors $\mathbf{t}_{i}$, $\mathbf{p}_{f}-\mathbf{p}_{i}, \mathbf{t}_{f}$ are nearly aligned, one might naturally expect the curve $\mathbf{r}(\xi)$ to be nearly linear. However, if $\left(\mathbf{u}_{f}, \mathbf{v}_{f}\right)$ differ markedly from $\left(\mathbf{u}_{i}, \mathbf{v}_{i}\right)$ in such cases, the accommodation of these disparate normal-plane vectors as initial/final instances of a rotation-minimizing frame will force $\mathbf{r}(\xi)$ to deviate markedly from linearity, since the angular speed of an RMF is equal to the curvature $\kappa(\xi)$. The possibility of constructing smooth motion interpolants is thus contingent on specifying end frames for which the difference between $\left(\mathbf{u}_{f}, \mathbf{v}_{f}\right)$ and $\left(\mathbf{u}_{i}, \mathbf{v}_{i}\right)$ is commensurate with that between $\mathbf{t}_{f}$ and $\mathbf{t}_{i}$.

Since the constraint that identifies the RRMF curves among all spatial PH quintics is a vector condition [6, 10] in $\mathbb{R}^{3}$, the two scalar freedoms $\phi_{0}, \phi_{2}$ of $C^{1} \mathrm{PH}$ quintic Hermite interpolants do not suffice to achieve its satisfaction. To construct RRMF quintics geometrically, we need to introduce further free parameters. A natural approach is to relax the Hermite data from $C^{1}$ to $G^{1}$, i.e., to impose end tangents $\mathbf{t}_{i}, \mathbf{t}_{f}$ instead of derivatives $\mathbf{d}_{i}, \mathbf{d}_{f}$ by setting $\mathbf{r}^{\prime}(0)=\ell_{0}^{2} \mathbf{t}_{i}, \mathbf{r}^{\prime}(1)=\ell_{2}^{2} \mathbf{t}_{f}$ for indeterminate (nonzero) scalars $\ell_{0}, \ell_{2}$.

The free parameters $\phi_{0}, \phi_{2}, \ell_{0}, \ell_{2}$ are then available to ensure satisfaction of the vector RRMF constraint, and the relative normal-plane orientation of the initial and final frames $\left(\mathbf{t}_{i}, \mathbf{u}_{i}, \mathbf{v}_{i}\right)$ and $\left(\mathbf{t}_{f}, \mathbf{u}_{f}, \mathbf{v}_{f}\right)$. Since computation of RMFs is an initial-value problem, an additional free parameter is available to make the absolute RMF orientation coincide with $\left(\mathbf{t}_{i}, \mathbf{u}_{i}, \mathbf{v}_{i}\right)$ initially, and it is shown that $\phi_{1}$ can be reintroduced for this purpose. Although there are nominally sufficient freedoms to match the interpolation conditions, the highly nonlinear nature of the problem makes it difficult to formulate simple a priori guarantees for existence of interpolants to arbitrary initial data.

It is shown that the problem is decomposable into four consecutive phases; namely, (1) interpolation of the tangents $\mathbf{t}_{i}$ and $\mathbf{t}_{f}$; (2) satisfaction of the RRMF constraint; (3) interpolation of the normal-plane vectors $\left(\mathbf{u}_{i}, \mathbf{v}_{i}\right)$ and $\left(\mathbf{u}_{f}, \mathbf{v}_{f}\right)$; and (4) interpolation of the end-point displacement $\mathbf{p}_{f}-\mathbf{p}_{i}$. Phases (1)-(3) possess closed-form algebraic solutions, and the difficulty of existence arises in phase (4). Since the solutions to phases (1)-(3) are independent of phase (4), it is possible to introduce new freedoms (e.g., through multiplying the hodograph by a scalar polynomial) in the latter phase that facilitate the existence of solutions. It is also shown that, for $G^{1}$ position and RMF data sampled asymptotically from a smooth analytic curve, RRMF quintic motion interpolants exist without the need for additional free parameters.

The remainder of the paper is organized as follows. After briefly reviewing the definition and basic properties of quintic RRMF curves in Section 2, the four phases of the $G^{1}$ RRMF quintic motion interpolation algorithm are developed in Section 3, A number of computed examples are then presented in Section 4, noting that the nominal interpolation process may not admit solutions for certain $G^{1}$ endpoint Hermite data. Section 5 then verifies the existence of solutions in the case of Hermite data sampled asymptotically from a smooth curve. Finally, Section 6 summarizes key results of the paper, and identifies open problems that deserve further investigation. 


\section{Characterization of RRMF quintics}

A polynomial Pythagorean-hodograph $(\mathrm{PH})$ curve $\mathbf{r}(\xi)=(x(\xi), y(\xi), z(\xi))$ is characterized by a derivative $\mathbf{r}^{\prime}(\xi)=\left(x^{\prime}(\xi), y^{\prime}(\xi), z^{\prime}(\xi)\right)$ satisfying

$$
x^{\prime 2}(\xi)+y^{\prime 2}(\xi)+z^{\prime 2}(\xi)=\sigma^{2}(\xi)
$$

for some polynomial $\sigma(\xi)$. The quaternion and Hopf map forms [3, 7] are two alternative (equivalent) models for the construction of $\mathrm{PH}$ curves. The former generates a Pythagorean hodograph $\mathbf{r}^{\prime}(\xi)$ from a quaternion polynomia

$$
\mathcal{A}(\xi)=u(\xi)+v(\xi) \mathbf{i}+p(\xi) \mathbf{j}+q(\xi) \mathbf{k}
$$

and its conjugate $\mathcal{A}^{*}(\xi)=u(\xi)-v(\xi) \mathbf{i}-p(\xi) \mathbf{j}-q(\xi) \mathbf{k}$ through the product

$$
\begin{aligned}
\mathbf{r}^{\prime}(\xi)=\mathcal{A}(\xi) & \mathbf{i} \mathcal{A}^{*}(\xi)=\left[u^{2}(\xi)+v^{2}(\xi)-p^{2}(\xi)-q^{2}(\xi)\right] \mathbf{i} \\
& +2[u(\xi) q(\xi)+v(\xi) p(\xi)] \mathbf{j}+2[v(\xi) q(\xi)-u(\xi) p(\xi)] \mathbf{k} .
\end{aligned}
$$

The latter generates a Pythagorean hodograph from complex polynomials

$$
\boldsymbol{\alpha}(\xi)=u(\xi)+\mathrm{i} v(\xi), \quad \boldsymbol{\beta}(\xi)=q(\xi)+\mathrm{i} p(\xi)
$$

through the expression

$$
\mathbf{r}^{\prime}(\xi)=\left(|\boldsymbol{\alpha}(\xi)|^{2}-|\boldsymbol{\beta}(\xi)|^{2}, 2 \operatorname{Re}(\boldsymbol{\alpha}(\xi) \overline{\boldsymbol{\beta}}(\xi)), 2 \operatorname{Im}(\boldsymbol{\alpha}(\xi) \overline{\boldsymbol{\beta}}(\xi))\right) .
$$

The equivalence of (4) and (5) can be verified by setting $\mathcal{A}(\xi)=\boldsymbol{\alpha}(\xi)+\mathbf{k} \boldsymbol{\beta}(\xi)$, the imaginary unit $\mathrm{i}$ being identified with the quaternion element $\mathbf{i}$. We find it convenient to simultaneously employ both the representations (4) and (5) of spatial $\mathrm{PH}$ curves (see [5] for a more thorough treatment of them).

A spatial PH quintic may be defined in terms of a quadratic quaternion polynomial, given in Bernstein form as,

$$
\mathcal{A}(\xi)=\mathcal{A}_{0}(1-\xi)^{2}+\mathcal{A}_{1} 2(1-\xi) \xi+\mathcal{A}_{2} \xi^{2},
$$

by substituting into (4) and integrating. This yields the Bézier form

$$
\mathbf{r}(\xi)=\sum_{i=0}^{5} \mathbf{p}_{i}\left(\begin{array}{l}
5 \\
i
\end{array}\right)(1-\xi)^{5-i} \xi^{i}
$$

of the $\mathrm{PH}$ quintic, with control points $\mathbf{p}_{i}=x_{i} \mathbf{i}+y_{i} \mathbf{j}+z_{i} \mathbf{k}$ given by

$$
\begin{aligned}
& \mathbf{p}_{1}=\mathbf{p}_{0}+\frac{1}{5} \mathcal{A}_{0} \mathbf{i} \mathcal{A}_{0}^{*}, \\
& \mathbf{p}_{2}=\mathbf{p}_{1}+\frac{1}{10}\left(\mathcal{A}_{0} \mathbf{i} \mathcal{A}_{1}^{*}+\mathcal{A}_{1} \mathbf{i} \mathcal{A}_{0}^{*}\right), \\
& \mathbf{p}_{3}=\mathbf{p}_{2}+\frac{1}{30}\left(\mathcal{A}_{0} \mathbf{i} \mathcal{A}_{2}^{*}+4 \mathcal{A}_{1} \mathbf{i} \mathcal{A}_{1}^{*}+\mathcal{A}_{2} \mathbf{i} \mathcal{A}_{0}^{*}\right), \\
& \mathbf{p}_{4}=\mathbf{p}_{3}+\frac{1}{10}\left(\mathcal{A}_{1} \mathbf{i} \mathcal{A}_{2}^{*}+\mathcal{A}_{2} \mathbf{i} \mathcal{A}_{1}^{*}\right), \\
& \mathbf{p}_{5}=\mathbf{p}_{4}+\frac{1}{5} \mathcal{A}_{2} \mathbf{i} \mathcal{A}_{2}^{*},
\end{aligned}
$$

where $\mathbf{p}_{0}$ is freely chosen. Alternatively, $\mathbf{r}(\xi)$ may be defined in terms of two complex quadratic polynomials, written in Bernstein form as

$$
\begin{gathered}
\boldsymbol{\alpha}(\xi)=\boldsymbol{\alpha}_{0}(1-\xi)^{2}+\boldsymbol{\alpha}_{1} 2(1-\xi) \xi+\boldsymbol{\alpha}_{2} \xi^{2}, \\
\boldsymbol{\beta}(\xi)=\boldsymbol{\beta}_{0}(1-\xi)^{2}+\boldsymbol{\beta}_{1} 2(1-\xi) \xi+\boldsymbol{\beta}_{2} \xi^{2},
\end{gathered}
$$

\footnotetext{
${ }^{2}$ Calligraphic characters such as $\mathcal{A}$ are used to denote quaternions, the scalar and vector parts being indicated by $\operatorname{scal}(\mathcal{A})$ and $\operatorname{vect}(\mathcal{A})$. Bold symbols denote complex numbers or vectors in $\mathbb{R}^{3}$; the meaning should be clear from the context.
} 
by substituting in (5) and integrating. Note that the quaternion and complex coefficients in (6) and (8) are related by $\mathcal{A}_{i}=\boldsymbol{\alpha}_{i}+\mathbf{k} \boldsymbol{\beta}_{i}$ for $i=0,1,2$.

Now the RRMF quintics form a proper subset of the spatial PH quintics. They may be characterized by constraints on the coefficients of the quaternion polynomial (6) or complex polynomials (8), defining the quaternion and Hopf map forms of spatial PH quintics, as follow: 3 ; see [6] for complete details.

Proposition 1. A PH quintic defined by the quadratic quaternion polynomial (6) has a rational $R M F$ if and only if the coefficients satisfy

$$
\mathcal{A}_{1} \mathbf{i} \mathcal{A}_{1}^{*}=\operatorname{vect}\left(\mathcal{A}_{2} \mathbf{i} \mathcal{A}_{0}^{*}\right) \text {. }
$$

Proposition 2. A PH quintic defined by the quadratic complex polynomials (8) has a rational $R M F$ if and only if the coefficients satisfy

$$
\operatorname{Re}\left(\boldsymbol{\alpha}_{0} \overline{\boldsymbol{\alpha}}_{2}-\boldsymbol{\beta}_{0} \overline{\boldsymbol{\beta}}_{2}\right)=\left|\boldsymbol{\alpha}_{1}\right|^{2}-\left|\boldsymbol{\beta}_{1}\right|^{2}, \quad \boldsymbol{\alpha}_{0} \overline{\boldsymbol{\beta}}_{2}+\boldsymbol{\alpha}_{2} \overline{\boldsymbol{\beta}}_{0}=2 \boldsymbol{\alpha}_{1} \overline{\boldsymbol{\beta}}_{1} .
$$

For applications such as swept surface construction and rigid body motion specification, an explicit rational representation of the RMF associated with any RRMF curve is desired. In terms of the quaternion representation, this can be conveniently expressed by invoking the Euler-Rodrigues frame (ERF) introduced by Choi and Han [2. For any spatial PH curve, the $\operatorname{ERF}(\mathbf{t}, \mathbf{f}, \mathbf{g})$ is a rational adapted frame defined by

$$
\mathbf{t}(\xi)=\frac{\mathcal{A}(\xi) \mathbf{i} \mathcal{A}^{*}(\xi)}{|\mathcal{A}(\xi)|^{2}}, \mathbf{f}(\xi)=\frac{\mathcal{A}(\xi) \mathbf{j} \mathcal{A}^{*}(\xi)}{|\mathcal{A}(\xi)|^{2}}, \mathbf{g}(\xi)=\frac{\mathcal{A}(\xi) \mathbf{k} \mathcal{A}^{*}(\xi)}{|\mathcal{A}(\xi)|^{2}} .
$$

Although the ERF is not ordinarily rotation-minimizing (see Figure1), it is a useful "reference" frame for the computation of RMFs.
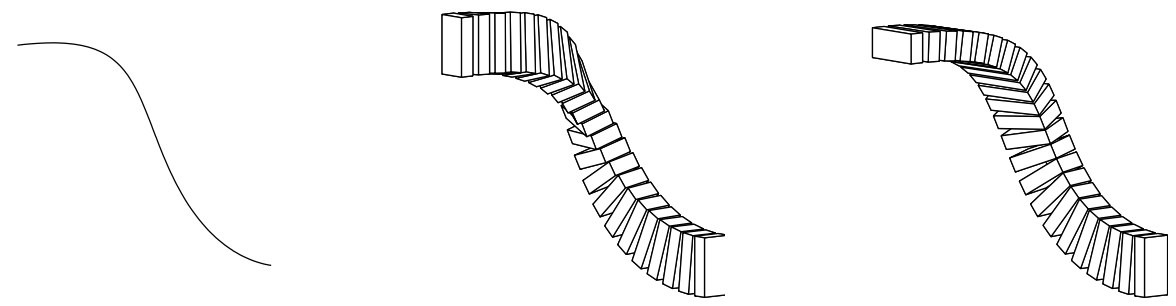

FiguRE 1. Left: trajectory for the spatial motion of a rigid body, specified by a quintic RRMF curve. The orientations of the body along the path, defined by the ERF (center) and the RMF (right) - coincident at the lower right - are also shown. One axis of the body remains aligned with the path tangent.

Han [17] showed that the $\mathrm{PH}$ curve defined through (4) by the quaternion polynomial $\mathcal{A}(\xi)=u(\xi)+v(\xi) \mathbf{i}+p(\xi) \mathbf{j}+q(\xi) \mathbf{k}$ admits a rational RMF if and only if polynomials $a(\xi), b(\xi)$ exist, with $\operatorname{gcd}(a(\xi), b(\xi))=$ constant, such that the components of $\mathcal{A}(\xi)$ satisfy

$$
\frac{u v^{\prime}-u^{\prime} v-p q^{\prime}+p^{\prime} q}{u^{2}+v^{2}+p^{2}+q^{2}}=\frac{a b^{\prime}-a^{\prime} b}{a^{2}+b^{2}} .
$$

\footnotetext{
${ }^{3}$ See also [16] for another characterization, in terms of a polynomial divisibility criterion.
} 
In terms of the Hopf map representation, this condition can be interpreted as requiring the existence of a complex polynomial $\mathbf{w}(\xi)=a(\xi)+\mathrm{i} b(\xi)$, with $\operatorname{gcd}(a(\xi), b(\xi))$ $=$ constant, such that

$$
\frac{\operatorname{Im}\left(\overline{\boldsymbol{\alpha}} \boldsymbol{\alpha}^{\prime}+\overline{\boldsymbol{\beta}} \boldsymbol{\beta}^{\prime}\right)}{|\boldsymbol{\alpha}|^{2}+|\boldsymbol{\beta}|^{2}}=\frac{\operatorname{Im}\left(\overline{\mathbf{w}} \mathbf{w}^{\prime}\right)}{|\mathbf{w}|^{2}} .
$$

For PH cubics, the condition (12) is satisfied [17] only in the degenerate case of planar curves, while for PH quintics Proposition 1 specifies a sufficient and necessary condition for its satisfaction. If (12) is satisfied, the RMF is obtained [17 from the ERF by a rational rotation of the normal-plane vectors $\mathbf{f}, \mathbf{g}$ at each curve point, specified in terms of the polynomials $a(\xi), b(\xi)$ by

$$
\begin{aligned}
& \mathbf{u}(\xi)=\frac{a^{2}(\xi)-b^{2}(\xi)}{a^{2}(\xi)+b^{2}(\xi)} \mathbf{f}(\xi)-\frac{2 a(\xi) b(\xi)}{a^{2}(\xi)+b^{2}(\xi)} \mathbf{g}(\xi), \\
& \mathbf{v}(\xi)=\frac{2 a(\xi) b(\xi)}{a^{2}(\xi)+b^{2}(\xi)} \mathbf{f}(\xi)+\frac{a^{2}(\xi)-b^{2}(\xi)}{a^{2}(\xi)+b^{2}(\xi)} \mathbf{g}(\xi)
\end{aligned}
$$

This is equivalent to writing

$$
\left[\begin{array}{l}
\mathbf{u}(\xi) \\
\mathbf{v}(\xi)
\end{array}\right]=\left[\begin{array}{rr}
\cos \theta(\xi) & -\sin \theta(\xi) \\
\sin \theta(\xi) & \cos \theta(\xi)
\end{array}\right]\left[\begin{array}{l}
\mathbf{f}(\xi) \\
\mathbf{g}(\xi)
\end{array}\right]
$$

for a rotation angle $\theta(\xi)$ in the curve normal plane at each point specified by

$$
\theta(\xi)=2 \arctan \frac{b(\xi)}{a(\xi)} .
$$

Note, however, that (15) and (16) do not uniquely define the RMF, since for a given curve the polynomials $a(\xi), b(\xi)$ satisfying (12) are not unique.

To clarify this, we note that the RMF can be more compactly expressed in quaternion form by setting

$$
\mathcal{W}(\xi)=a(\xi)+b(\xi) \mathbf{i},
$$

for which we have

$$
\begin{aligned}
\mathcal{W}^{*}(\xi) \mathbf{i} \mathcal{W}(\xi) & =\left[\left(a^{2}(\xi)+b^{2}(\xi)\right] \mathbf{i},\right. \\
\mathcal{W}^{*}(\xi) \mathbf{j} \mathcal{W}(\xi) & =\left[\left(a^{2}(\xi)-b^{2}(\xi)\right] \mathbf{j}-2 a(\xi) b(\xi) \mathbf{k},\right. \\
\mathcal{W}^{*}(\xi) \mathbf{k} \mathcal{W}(\xi) & =2 a(\xi) b(\xi) \mathbf{j}+\left[a^{2}(\xi)-b^{2}(\xi)\right] \mathbf{k} .
\end{aligned}
$$

Hence, by writing

$$
\mathcal{B}(\xi)=\mathcal{A}(\xi) \mathcal{W}^{*}(\xi),
$$

the RMF can be expressed as

$$
\mathbf{t}(\xi)=\frac{\mathcal{B}(\xi) \mathbf{i} \mathcal{B}^{*}(\xi)}{|\mathcal{B}(\xi)|^{2}}, \quad \mathbf{u}(\xi)=\frac{\mathcal{B}(\xi) \mathbf{j} \mathcal{B}^{*}(\xi)}{|\mathcal{B}(\xi)|^{2}}, \quad \mathbf{v}(\xi)=\frac{\mathcal{B}(\xi) \mathbf{k} \mathcal{B}^{*}(\xi)}{|\mathcal{B}(\xi)|^{2}} .
$$

In terms of the quaternion polynomials $\mathcal{A}(\xi)$ and $\mathcal{W}(\xi)$, the RRMF condition (12) can be written as

$$
\frac{\operatorname{scal}\left(\mathcal{A}(\xi) \mathbf{i} \mathcal{A}^{\prime *}(\xi)\right)}{|\mathcal{A}(\xi)|^{2}}=\frac{\operatorname{scal}\left(\mathcal{W}(\xi) \mathbf{i} \mathcal{W}^{\prime *}(\xi)\right)}{|\mathcal{W}(\xi)|^{2}}
$$


Remark 1. If a spatial $\mathrm{PH}$ curve $\mathbf{r}(\xi)$ defined by the hodograph (4) satisfies the RRMF condition (20), then the spatial PH curve $\tilde{\mathbf{r}}(\xi)$ defined by replacing the quaternion polynomial $\mathcal{A}(\xi)$ with

$$
\tilde{\mathcal{A}}(\xi)=\mathcal{A}(\xi) \exp \left(\frac{1}{2} \psi \mathbf{i}\right)
$$

also satisfies (20) for the same $\mathcal{W}(\xi)$. The curve $\tilde{\mathbf{r}}(\xi)$ is identical to $\mathbf{r}(\xi)$, but has a different Euler-Rodrigues frame, specified by $\tilde{\mathbf{t}}(\xi)=\mathbf{t}(\xi)$ and

$$
\left[\begin{array}{c}
\tilde{\mathbf{f}}(\xi) \\
\tilde{\mathbf{g}}(\xi)
\end{array}\right]=\left[\begin{array}{rr}
\cos \psi & \sin \psi \\
-\sin \psi & \cos \psi
\end{array}\right]\left[\begin{array}{c}
\mathbf{f}(\xi) \\
\mathbf{g}(\xi)
\end{array}\right]
$$

i.e., the normal-plane ERF vectors $\tilde{\mathbf{f}}(\xi), \tilde{\mathbf{g}}(\xi)$ of $\tilde{\mathbf{r}}(\xi)$ exhibit a fixed angular displacement $\psi$ relative to the corresponding vectors $\mathbf{f}(\xi), \mathbf{g}(\xi)$ of $\mathbf{r}(\xi)$. From (15), we see that the normal-plane RMF vectors $\tilde{\mathbf{u}}(\xi), \tilde{\mathbf{v}}(\xi)$ of $\tilde{\mathbf{r}}(\xi)$ have the same fixed angle $\psi$ relative to the corresponding vectors $\mathbf{u}(\xi), \mathbf{v}(\xi)$ of $\mathbf{r}(\xi)$.

The free parameter $\psi$ in (21) can be invoked to achieve any desired initial orientation of the RMF on a given curve $\mathbf{r}(\xi)$. This freedom reflects the fact that computing RMFs on a curve corresponds to an initial-value problem.

\section{RRMF QUINTIC MOTION INTERPOLANTS}

A rational rigid-body motion along a prescribed path $\mathbf{r}(\xi)$ in $\mathbb{R}^{3}$ is specified by a rational orthonormal frame $(\mathbf{t}(\xi), \mathbf{u}(\xi), \mathbf{v}(\xi))$ that defines the orientation of the body as it executes the prescribed path. The frame is said to be adapted to $\mathbf{r}(\xi)$ if the first frame vector coincides with the curve tangent, i.e., $\mathbf{t}=\mathbf{r}^{\prime} /\left|\mathbf{r}^{\prime}\right|$, and it is rotation-minimizing when its angular velocity $\boldsymbol{\omega}$ has no component in the direction of $\mathbf{t}$, i.e., $\boldsymbol{\omega} \cdot \mathbf{t} \equiv 0$ so that $\mathbf{u}$ and $\mathbf{v}$ exhibit no instantaneous rotation about $\mathbf{t}$ (equivalently, the derivatives $\mathbf{u}^{\prime}$ and $\mathbf{v}^{\prime}$ are parallel to $\mathbf{t}$ ).

We wish to construct RRMF quintics $\mathbf{r}(\xi)$ for $\xi \in[0,1]$ that interpolate given initial/final points $\mathbf{p}_{i}$ and $\mathbf{p}_{f}$, and instances $\left(\mathbf{t}_{i}, \mathbf{u}_{i}, \mathbf{v}_{i}\right)$ and $\left(\mathbf{t}_{f}, \mathbf{u}_{f}, \mathbf{v}_{f}\right)$ of the rotationminimizing frame. Interpolation of the end points and tangents implies that

$$
\mathbf{r}(0)=\mathbf{p}_{i}, \quad \frac{\mathbf{r}^{\prime}(0)}{\left|\mathbf{r}^{\prime}(0)\right|}=\mathbf{t}_{i} \quad \text { and } \quad \mathbf{r}(1)=\mathbf{p}_{f}, \quad \frac{\mathbf{r}^{\prime}(1)}{\left|\mathbf{r}^{\prime}(1)\right|}=\mathbf{t}_{f} .
$$

The components of the tangents are denoted by

$$
\mathbf{t}_{i}=\left(\lambda_{i}, \mu_{i}, \nu_{i}\right) \quad \text { and } \quad \mathbf{t}_{f}=\left(\lambda_{f}, \mu_{f}, \nu_{f}\right) .
$$

Interpolation of the prescribed normal-plane vectors $\mathbf{u}_{i}, \mathbf{v}_{i}$ and $\mathbf{u}_{f}, \mathbf{v}_{f}$ at the curve end points will be addressed in Section 3.3. To simplify the analysis we may assume, without loss of generality, that the displacement $\Delta \mathbf{p}=\mathbf{p}_{f}-\mathbf{p}_{i}$ is in the positive $x$-direction, and set $\Delta \mathbf{p}=L \mathbf{i}$. We also restrict our attention to nonplanar data, characterized by the fact that $\mathbf{i} \cdot\left(\mathbf{t}_{i} \times \mathbf{t}_{f}\right) \neq 0$.

Recall [9] that, for a given vector $\mathbf{d}$, the quaternion equation

$$
\mathcal{A} \text { i } \mathcal{A}^{*}=\mathrm{d}
$$

has the one-parameter family of solutions defined 4 by

$$
\mathcal{A}=\sqrt{d} \mathbf{n} \exp (\phi \mathbf{i})
$$

\footnotetext{
${ }^{4}$ Note that, in expressions juxtaposing scalars, vectors, and quaternions, such as (23), the quaternion product is always imputed.
} 
where $d=|\mathbf{d}|, \phi$ is a free parameter, $\exp (\phi \mathbf{i})=\cos \phi+\sin \phi \mathbf{i}$, and

$$
\mathbf{n}=\frac{d \mathbf{i}+\mathbf{d}}{|d \mathbf{i}+\mathbf{d}|}
$$

is the unit bisector of $\mathbf{i}$ and a unit vector in the direction of $\mathbf{d}$. In the singular case $\mathbf{d}=-d \mathbf{i}$, the solution to (22) becomes

$$
\mathcal{A}=\sqrt{d}\left(\mathbf{e}_{1} \cos \phi+\mathbf{e}_{2} \sin \phi\right),
$$

where $\mathbf{e}_{1}, \mathbf{e}_{2}$ are two unit vectors orthogonal to $\mathbf{i}$ and each other. For brevity, we do not explicitly reiterate this special case in subsequent applications of the general solution (23) to equation (22).

3.1. Interpolation of end tangents. From (4) and (6), interpolation of the end tangents yields the equations

$$
\frac{\mathcal{A}_{0} \mathbf{i} \mathcal{A}_{0}^{*}}{\left|\mathcal{A}_{0}\right|^{2}}=\mathbf{t}_{i} \quad \text { and } \quad \frac{\mathcal{A}_{2} \mathbf{i} \mathcal{A}_{2}^{*}}{\left|\mathcal{A}_{2}\right|^{2}}=\mathbf{t}_{f},
$$

which are essentially of the form (22). Hence, the solutions can be expressed in terms of free parameters $\ell_{0}, \ell_{2}$ and $\phi_{0}, \phi_{2}$ as

$$
\mathcal{A}_{0}=\ell_{0} \mathbf{n}_{0} \exp \left(\phi_{0} \mathbf{i}\right) \quad \text { and } \quad \mathcal{A}_{2}=\ell_{2} \mathbf{n}_{2} \exp \left(\phi_{2} \mathbf{i}\right)
$$

where $\mathbf{n}_{0}$ and $\mathbf{n}_{2}$ are the unit bisectors of $\mathbf{i}$ with $\mathbf{t}_{i}$ and $\mathbf{t}_{f}$, defined by

$$
\mathbf{n}_{0}=\frac{\mathbf{i}+\mathbf{t}_{i}}{\left|\mathbf{i}+\mathbf{t}_{i}\right|} \quad \text { and } \quad \mathbf{n}_{2}=\frac{\mathbf{i}+\mathbf{t}_{f}}{\left|\mathbf{i}+\mathbf{t}_{f}\right|} .
$$

Note that $\mathbf{i}, \mathbf{n}_{0}, \mathbf{n}_{2}$ are linearly independent if and only if $\mathbf{i}, \mathbf{t}_{i}, \mathbf{t}_{f}$ are linearly independent, since $\mathbf{i} \cdot\left(\mathbf{n}_{0} \times \mathbf{n}_{2}\right)=\mathbf{i} \cdot\left(\mathbf{t}_{i} \times \mathbf{t}_{f}\right) /\left(\left|\mathbf{i}+\mathbf{t}_{i}\right|\left|\mathbf{i}+\mathbf{t}_{f}\right|\right)$.

3.2. Satisfaction of RRMF constraint. Substituting from (24) and using [23] the scalar-vector form

$$
\mathcal{A B}=(a b-\mathbf{a} \cdot \mathbf{b}, a \mathbf{b}+b \mathbf{a}+\mathbf{a} \times \mathbf{b})
$$

for the product of two quaternions $\mathcal{A}=(a, \mathbf{a})$ and $\mathcal{B}=(b, \mathbf{b})$, the quantity $\operatorname{vect}\left(\mathcal{A}_{2} \mathbf{i} \mathcal{A}_{0}^{*}\right)=\frac{1}{2}\left(\mathcal{A}_{2} \mathbf{i} \mathcal{A}_{0}^{*}+\mathcal{A}_{0} \mathbf{i} \mathcal{A}_{2}^{*}\right)$, required in subsequent steps of the interpolation scheme, can be expressed in terms of $\ell_{0}, \ell_{2}$ and $\phi_{0}, \phi_{2}$ as

$$
\frac{1}{2}\left(\mathcal{A}_{2} \mathbf{i} \mathcal{A}_{0}^{*}+\mathcal{A}_{0} \mathbf{i} \mathcal{A}_{2}^{*}\right)=\ell_{0} \ell_{2}\left[\mathbf{x} \cos \left(\phi_{2}-\phi_{0}\right)+\mathbf{y} \sin \left(\phi_{2}-\phi_{0}\right)\right],
$$

where we define

$$
\mathbf{x}=\left(\mathbf{i} \cdot \mathbf{n}_{2}\right) \mathbf{n}_{0}+\left(\mathbf{i} \cdot \mathbf{n}_{0}\right) \mathbf{n}_{2}-\left(\mathbf{n}_{0} \cdot \mathbf{n}_{2}\right) \mathbf{i}, \quad \mathbf{y}=\mathbf{n}_{2} \times \mathbf{n}_{0} .
$$

Now the RRMF condition (9) is equivalent to

$$
\mathcal{A}_{1} \mathbf{i} \mathcal{A}_{1}^{*}=\frac{1}{2}\left(\mathcal{A}_{0} \mathbf{i} \mathcal{A}_{2}^{*}+\mathcal{A}_{2} \mathbf{i} \mathcal{A}_{0}^{*}\right),
$$

and using (27), it can be expressed as

$$
\mathcal{A}_{1} \text { i } \mathcal{A}_{1}^{*}=\ell_{0} \ell_{2} \mathbf{z},
$$

where

$$
\mathbf{z}=\mathbf{x} \cos \left(\phi_{2}-\phi_{0}\right)+\mathbf{y} \sin \left(\phi_{2}-\phi_{0}\right) .
$$

As in [9], it is convenient to write

$$
\alpha=\frac{1}{2}\left(\phi_{0}+\phi_{2}\right) \quad \text { and } \quad \beta=\phi_{2}-\phi_{0} .
$$


The vector (30) traces an ellipse in the plane spanned by $\mathbf{x}, \mathbf{y}$ as $\beta=\phi_{2}-\phi_{0}$ varies. Its magnitude is given by

$$
z=|\mathbf{z}|=\sqrt{|\mathbf{x}|^{2} \cos ^{2} \beta+2 \mathbf{x} \cdot \mathbf{y} \cos \beta \sin \beta+|\mathbf{y}|^{2} \sin ^{2} \beta} .
$$

By writing

$$
\gamma=\mathbf{i} \cdot\left(\mathbf{n}_{2} \times \mathbf{n}_{0}\right), \quad \delta=\mathbf{n}_{0} \cdot \mathbf{n}_{2}
$$

and observing that

$$
\left|\left(\mathbf{i} \cdot \mathbf{n}_{2}\right) \mathbf{n}_{0}+\left(\mathbf{i} \cdot \mathbf{n}_{0}\right) \mathbf{n}_{2}-\left(\mathbf{n}_{0} \cdot \mathbf{n}_{2}\right) \mathbf{i}\right|^{2}=1-\left[\mathbf{i} \cdot\left(\mathbf{n}_{2} \times \mathbf{n}_{0}\right)\right]^{2},
$$

we obtain

$$
|\mathbf{x}|^{2}=1-\gamma^{2}, \quad \mathbf{x} \cdot \mathbf{y}=-\gamma \delta, \quad|\mathbf{y}|^{2}=1-\delta^{2},
$$

and hence (32) can be expressed as

$$
z=\sqrt{1-(\gamma \cos \beta+\delta \sin \beta)^{2}} .
$$

Since equation (29) is of the form (22), its solution can be written [9] as

$$
\mathcal{A}_{1}=\sqrt{\ell_{0} \ell_{2} z} \mathbf{n}_{1} \exp \left(\phi_{1} \mathbf{i}\right),
$$

where $\phi_{1}$ is a free parameter, and

$$
\mathbf{n}_{1}=\frac{z \mathbf{i}+\mathbf{z}}{|z \mathbf{i}+\mathbf{z}|}
$$

is the unit bisector of $\mathbf{i}$ and a unit vector in the direction of $\mathbf{z}$.

In prior studies [8, 9] of Hermite interpolation with spatial PH quintics, it was noted that the interpolants depend only on the differences of the angular parameters $\phi_{0}, \phi_{1}, \phi_{2}$ occurring in (24) and (36), and thus one may set $\phi_{1}=0$ (say) without loss of generality. In the present context, however, we observed in Remark 1 the importance of the nonuniqueness of the polynomial $\mathcal{A}(\xi)$ in facilitating interpolation of the initial frame $\left(\mathbf{t}_{i}, \mathbf{u}_{i}, \mathbf{v}_{i}\right)$.

The free parameter $\psi$ in the family (21) of quaternion polynomials that yield exactly the same hodograph $\mathbf{r}^{\prime}(\xi)$ on substituting $\tilde{\mathcal{A}}(\xi)$ for $\mathcal{A}(\xi)$ in (4) is equivalent to retaining $\phi_{1}$ as a free parameter in (36), rather than imposing the customary choice $\phi_{1}=0$ for Hermite interpolation of end points/tangents $\mathbf{p}_{i}, \mathbf{p}_{f}$ and $\mathbf{t}_{i}, \mathbf{t}_{f}$ without reference to the frame vectors $\mathbf{u}_{i}, \mathbf{v}_{i}$ and $\mathbf{u}_{f}, \mathbf{v}_{f}$. We therefore retain $\phi_{1}$ as a free parameter, for use in the interpolation scheme.

3.3. Interpolation of normal-plane vectors. The function (16) specifying the orientation of the RMF relative to the ERF is defined in terms of the polynomials $a(\xi), b(\xi)$, i.e., the components of the quaternion polynomial (17). Identifying the quaternion element $\mathbf{i}$ with the imaginary unit i, we may regard (17) as a complex polynomia 5 . In the present context, we take it to be the quadratic

$$
\mathbf{w}(\xi)=a(\xi)+\mathrm{i} b(\xi)=\mathbf{w}_{0}(1-\xi)^{2}+\mathbf{w}_{1} 2(1-\xi) \xi+\mathbf{w}_{2} \xi^{2}
$$

\footnotetext{
${ }^{5}$ Quaternion polynomials whose $\mathbf{j}$, $\mathbf{k}$ components vanish identically can be interpreted as complex polynomials, the algebra of complex numbers being regarded as a (commutative) subalgebra of the quaternion algebra.
} 
with $\mathbf{w}_{i}=a_{i}+\mathrm{i} b_{i}$ for $i=0,1,2$, where $a_{0}, a_{1}, a_{2}$ and $b_{0}, b_{1}, b_{2}$ are the Bernstein coefficients of the real quadratic polynomials $a(\xi)$ and $b(\xi)$. Expression (16) can then be written as

$$
\theta(\xi)=2 \arg (\mathbf{w}(\xi))
$$

It was shown in [10] that, for an RRMF quintic defined in the Hopf map form (5) by the complex quadratic polynomials (8) satisfying (13), an instance of (38) can be specified in terms of the coefficients of $\boldsymbol{\alpha}(\xi), \boldsymbol{\beta}(\xi)$ as 6

$$
\mathbf{w}_{0}=1, \quad \mathbf{w}_{1}=\frac{\overline{\boldsymbol{\alpha}}_{0} \boldsymbol{\alpha}_{1}+\overline{\boldsymbol{\beta}}_{0} \boldsymbol{\beta}_{1}}{\left|\boldsymbol{\alpha}_{0}\right|^{2}+\left|\boldsymbol{\beta}_{0}\right|^{2}}, \quad \mathbf{w}_{2}=\frac{\overline{\boldsymbol{\alpha}}_{1} \boldsymbol{\alpha}_{2}+\overline{\boldsymbol{\beta}}_{1} \boldsymbol{\beta}_{2}}{\boldsymbol{\alpha}_{0} \overline{\boldsymbol{\alpha}}_{1}+\boldsymbol{\beta}_{0} \overline{\boldsymbol{\beta}}_{1}} .
$$

Through (38) and (39), these complex values completely specify the rational rotation (15) of the ERF onto the RMF.

Since the RMF is defined relative to the ERF, we need to determine the initial and final orientations of the latter. From (6), (11), and (24), the ERF normal plane vectors at $\xi=0$ and 1 can be written as

$$
\begin{array}{ll}
\mathbf{f}(0)=\cos 2 \phi_{0} \mathbf{j}_{0}+\sin 2 \phi_{0} \mathbf{k}_{0}, & \mathbf{g}(0)=\cos 2 \phi_{0} \mathbf{k}_{0}-\sin 2 \phi_{0} \mathbf{j}_{0}, \\
\mathbf{f}(1)=\cos 2 \phi_{2} \mathbf{j}_{2}+\sin 2 \phi_{2} \mathbf{k}_{2}, & \mathbf{g}(1)=\cos 2 \phi_{2} \mathbf{k}_{2}-\sin 2 \phi_{2} \mathbf{j}_{2},
\end{array}
$$

where, in terms of the unit vectors $\mathbf{n}_{0}$ and $\mathbf{n}_{2}$ specified by (25), we define

$$
\begin{array}{ll}
\mathbf{j}_{0}=2\left(\mathbf{j} \cdot \mathbf{n}_{0}\right) \mathbf{n}_{0}-\mathbf{j}, & \mathbf{k}_{0}=2\left(\mathbf{k} \cdot \mathbf{n}_{0}\right) \mathbf{n}_{0}-\mathbf{k}, \\
\mathbf{j}_{2}=2\left(\mathbf{j} \cdot \mathbf{n}_{2}\right) \mathbf{n}_{2}-\mathbf{j}, & \mathbf{k}_{2}=2\left(\mathbf{k} \cdot \mathbf{n}_{2}\right) \mathbf{n}_{2}-\mathbf{k} .
\end{array}
$$

These are simply the reflections of $\mathbf{j}$ and $\mathbf{k}$ in $\mathbf{n}_{0}$ and $\mathbf{n}_{2}$.

To impose the desired initial and final instances of the RMF it suffices to consider the vector $\mathbf{u}(\xi)$, since $\mathbf{v}(\xi)=\mathbf{t}(\xi) \times \mathbf{u}(\xi)$. From (15), the initial and final instances of $\mathbf{u}(\xi)$ are given by

$$
\begin{aligned}
& \mathbf{u}(0)=\cos \theta(0) \mathbf{f}(0)-\sin \theta(0) \mathbf{g}(0), \\
& \mathbf{u}(1)=\cos \theta(1) \mathbf{f}(1)-\sin \theta(1) \mathbf{g}(1),
\end{aligned}
$$

and on substituting from (41) and equating to $\mathbf{u}_{i}, \mathbf{u}_{f}$ these can be written as

$$
\begin{aligned}
& \cos \left(2 \phi_{0}-\theta(0)\right) \mathbf{j}_{0}+\sin \left(2 \phi_{0}-\theta(0)\right) \mathbf{k}_{0}=\mathbf{u}_{i}, \\
& \cos \left(2 \phi_{2}-\theta(1)\right) \mathbf{j}_{2}+\sin \left(2 \phi_{2}-\theta(1)\right) \mathbf{k}_{2}=\mathbf{u}_{f} .
\end{aligned}
$$

In the first equation we set $\theta(0)=2 \arg \left(\mathbf{w}_{0}\right)=0$ from (38)-(40). Taking dot products with $\mathbf{u}_{i}$ and $\mathbf{v}_{i}$, and noting that

$$
\left(\mathbf{j}_{0} \cdot \mathbf{u}_{i}\right)\left(\mathbf{k}_{0} \cdot \mathbf{v}_{i}\right)-\left(\mathbf{k}_{0} \cdot \mathbf{u}_{i}\right)\left(\mathbf{j}_{0} \cdot \mathbf{v}_{i}\right)=\left(\mathbf{j}_{0} \times \mathbf{k}_{0}\right) \cdot\left(\mathbf{u}_{i} \times \mathbf{v}_{i}\right)=\left|\mathbf{t}_{i}\right|^{2}=1,
$$

two $\phi_{0}$ values (differing by $\pi$ ) are determined from

$$
\cos 2 \phi_{0}=\mathbf{k}_{0} \cdot \mathbf{v}_{i} \quad \text { and } \quad \sin 2 \phi_{0}=-\mathbf{j}_{0} \cdot \mathbf{v}_{i} .
$$

Similarly, the dot products of the second equation in (43) with $\mathbf{u}_{f}, \mathbf{v}_{f}$ yield

$$
\cos \left(2 \phi_{2}-\theta(1)\right)=\mathbf{k}_{2} \cdot \mathbf{v}_{f} \quad \text { and } \quad \sin \left(2 \phi_{2}-\theta(1)\right)=-\mathbf{j}_{2} \cdot \mathbf{v}_{f}
$$

where $\theta(1)=2 \arg \left(\mathbf{w}_{2}\right)$. Now by introducing a new variable $\eta$ and writing

$$
\frac{\mathbf{w}_{2}}{\left|\mathbf{w}_{2}\right|}=\mathrm{e}^{\mathrm{i}\left(\phi_{2}-\eta\right)}
$$

\footnotetext{
${ }^{6}$ Although simpler expressions for $\mathbf{w}_{0}, \mathbf{w}_{1}, \mathbf{w}_{2}$ were derived in [6], they have not proved advantageous in the context of the problem under consideration.
} 
we have $\theta(1)=2\left(\phi_{2}-\eta\right)$. Hence, two $\eta$ values (differing by $\pi$ ) are determined from

$$
\cos 2 \eta=\mathbf{k}_{2} \cdot \mathbf{v}_{f} \quad \text { and } \quad \sin 2 \eta=-\mathbf{j}_{2} \cdot \mathbf{v}_{f} .
$$

It remains to fix the relation between $\phi_{2}$ and $\eta$. Writing $\mathbf{w}_{2}$ from (40) as

$$
\mathbf{w}_{2}=\frac{\overline{\boldsymbol{\alpha}}_{1} \boldsymbol{\alpha}_{2}+\overline{\boldsymbol{\beta}}_{1} \boldsymbol{\beta}_{2}}{\boldsymbol{\alpha}_{0} \overline{\boldsymbol{\alpha}}_{1}+\boldsymbol{\beta}_{0} \overline{\boldsymbol{\beta}}_{1}}=\frac{\left(\overline{\boldsymbol{\alpha}}_{1} \boldsymbol{\alpha}_{2}+\overline{\boldsymbol{\beta}}_{1} \boldsymbol{\beta}_{2}\right)\left(\overline{\boldsymbol{\alpha}}_{0} \boldsymbol{\alpha}_{1}+\overline{\boldsymbol{\beta}}_{0} \boldsymbol{\beta}_{1}\right)}{\left|\boldsymbol{\alpha}_{0} \overline{\boldsymbol{\alpha}}_{1}+\boldsymbol{\beta}_{0} \overline{\boldsymbol{\beta}}_{1}\right|^{2}},
$$

we have

$$
\mathrm{e}^{\mathrm{i}\left(\phi_{2}-\eta\right)}=\frac{\left(\overline{\boldsymbol{\alpha}}_{1} \boldsymbol{\alpha}_{2}+\overline{\boldsymbol{\beta}}_{1} \boldsymbol{\beta}_{2}\right)\left(\overline{\boldsymbol{\alpha}}_{0} \boldsymbol{\alpha}_{1}+\overline{\boldsymbol{\beta}}_{0} \boldsymbol{\beta}_{1}\right)}{\left|\left(\overline{\boldsymbol{\alpha}}_{1} \boldsymbol{\alpha}_{2}+\overline{\boldsymbol{\beta}}_{1} \boldsymbol{\beta}_{2}\right)\left(\overline{\boldsymbol{\alpha}}_{0} \boldsymbol{\alpha}_{1}+\overline{\boldsymbol{\beta}}_{0} \boldsymbol{\beta}_{1}\right)\right|} .
$$

It can be shown (see Appendix 1) that this reduces to the quadratic equation

$$
\left(1-\gamma^{2}-\delta^{2}\right)\left(c_{2} \tan ^{2} \beta+c_{1} \tan \beta+c_{0}\right)=0
$$

in $\tan \beta$, where $\beta=\phi_{2}-\phi_{0}$ and we define

$$
c_{0}=\gamma^{2}\left(t^{2}+1\right)-t^{2}, \quad c_{1}=2\left(\gamma \delta\left(t^{2}+1\right)-t\right), \quad c_{2}=\delta^{2}\left(t^{2}+1\right)-1,
$$

with $t=\tan \left(\phi_{0}-\eta\right)$ and $\gamma, \delta$ specified by (33). One can verify that

$$
1-\gamma^{2}-\delta^{2}=\frac{1}{2}\left(1-\mathbf{t}_{i} \cdot \mathbf{t}_{f}\right)
$$

which is evidently positive when $\mathbf{t}_{i}, \mathbf{t}_{f}$ are nonparallel (i.e., the Hermite data are nonplanar). Thus, discarding this factor, we find that the discriminant of (48) admits the nonnegative factorization

$$
\Delta=4\left(t^{2}+1\right)(\delta t-\gamma)^{2},
$$

and hence (48) always has real solutions, given by

$$
\beta=\arctan \frac{t-\gamma \delta\left(t^{2}+1\right) \pm(\delta t-\gamma) \sqrt{t^{2}+1}}{\delta^{2}\left(t^{2}+1\right)-1} .
$$

Due to the sign choice and multi-valued nature of the arctangent, this gives four values of $\beta=\phi_{2}-\phi_{0}$, modulo $2 \pi$. Since (48) was derived by squaring and taking tangents, however, only one value satisfies (47). It can be identified by checking that equations (71)-(72) in Appendix 1 are satisfied. From the correct $\beta$ value and known $\phi_{0}$ value, we obtain $\phi_{2}=\beta+\phi_{0}$.

The above procedure yields four possible $\left(\phi_{0}, \phi_{2}\right)$ pairs, of the form

$$
\left(\phi_{k}, \phi_{l}\right), \quad\left(\phi_{k}+\pi, \phi_{l}+\pi\right), \quad\left(\phi_{k}, \phi_{m}\right), \quad\left(\phi_{k}+\pi, \phi_{m}+\pi\right) .
$$

3.4. Interpolation of displacement. Choosing $\mathbf{p}_{i}$ as integration constant upon integrating the hodograph (4), and writing $\Delta \mathbf{p}=\mathbf{p}_{f}-\mathbf{p}_{i}$, interpolation of the end points yields the condition

$$
\begin{aligned}
\int_{0}^{1} \mathcal{A}(\xi) \mathbf{i} \mathcal{A}^{*}(\xi) \mathrm{d} \xi & =\frac{1}{5} \mathcal{A}_{0} \mathbf{i} \mathcal{A}_{0}^{*}+\frac{1}{10}\left(\mathcal{A}_{0} \mathbf{i} \mathcal{A}_{1}^{*}+\mathcal{A}_{1} \mathbf{i} \mathcal{A}_{0}^{*}\right) \\
& +\frac{1}{30}\left(\mathcal{A}_{0} \mathbf{i} \mathcal{A}_{2}^{*}+4 \mathcal{A}_{1} \mathbf{i} \mathcal{A}_{1}^{*}+\mathcal{A}_{2} \mathbf{i} \mathcal{A}_{0}^{*}\right) \\
& +\frac{1}{10}\left(\mathcal{A}_{1} \mathbf{i} \mathcal{A}_{2}^{*}+\mathcal{A}_{2} \mathbf{i} \mathcal{A}_{1}^{*}\right)+\frac{1}{5} \mathcal{A}_{2} \mathbf{i} \mathcal{A}_{2}^{*}=\Delta \mathbf{p} .
\end{aligned}
$$

Without loss of generality, we may set $\Delta \mathbf{p}=L \mathbf{i}$. Then writing $\mathcal{A}_{0} \mathbf{i} \mathcal{A}_{0}^{*}=\ell_{0}^{2} \mathbf{t}_{i}$, $\mathcal{A}_{2} \mathbf{i} \mathcal{A}_{2}^{*}=\ell_{0}^{2} \mathbf{t}_{f}$, and using (28) and (29), equation (51) reduces to

$$
\left(\mathcal{A}_{0}+\mathcal{A}_{2}\right) \mathbf{i} \mathcal{A}_{1}^{*}+\mathcal{A}_{1} \mathbf{i}\left(\mathcal{A}_{0}^{*}+\mathcal{A}_{2}^{*}\right)+2\left(\ell_{0}^{2} \mathbf{t}_{i}+\ell_{2}^{2} \mathbf{t}_{f}\right)+2 \ell_{0} \ell_{2} \mathbf{z}=10 L \mathbf{i} .
$$


Since $\phi_{0}, \phi_{2}$ (and $\beta=\phi_{2}-\phi_{0}$ ) have already been determined in Section 3.3 , the quaternions (24) and the vectors (30) and (37) are known quantities, so this is a vector equation in the three remaining parameters $\ell_{0}, \ell_{2}, \phi_{1}$.

Substituting from (24) and (36), equation (52) becomes

$$
\begin{aligned}
\sqrt{\ell_{0} \ell_{2} z}\left[\left(\ell_{0} \mathbf{a}_{0}+\ell_{2} \mathbf{a}_{2}\right) \cos \phi_{1}+\left(\ell_{0} \mathbf{b}_{0}\right.\right. & \left.\left.+\ell_{2} \mathbf{b}_{2}\right) \sin \phi_{1}\right] \\
& +\ell_{0}^{2} \mathbf{t}_{i}+\ell_{2}^{2} \mathbf{t}_{f}+\ell_{0} \ell_{2} \mathbf{z}=5 L \mathbf{i},
\end{aligned}
$$

where we define

$$
\begin{aligned}
\mathbf{a}_{0} & =\left[\left(\mathbf{i} \cdot \mathbf{n}_{0}\right) \mathbf{n}_{1}+\left(\mathbf{i} \cdot \mathbf{n}_{1}\right) \mathbf{n}_{0}-\left(\mathbf{n}_{0} \cdot \mathbf{n}_{1}\right) \mathbf{i}\right] \cos \phi_{0}+\mathbf{n}_{0} \times \mathbf{n}_{1} \sin \phi_{0}, \\
\mathbf{b}_{0} & =\left[\left(\mathbf{i} \cdot \mathbf{n}_{0}\right) \mathbf{n}_{1}+\left(\mathbf{i} \cdot \mathbf{n}_{1}\right) \mathbf{n}_{0}-\left(\mathbf{n}_{0} \cdot \mathbf{n}_{1}\right) \mathbf{i}\right] \sin \phi_{0}-\mathbf{n}_{0} \times \mathbf{n}_{1} \cos \phi_{0}, \\
\mathbf{a}_{2} & =\left[\left(\mathbf{i} \cdot \mathbf{n}_{2}\right) \mathbf{n}_{1}+\left(\mathbf{i} \cdot \mathbf{n}_{1}\right) \mathbf{n}_{2}-\left(\mathbf{n}_{2} \cdot \mathbf{n}_{1}\right) \mathbf{i}\right] \cos \phi_{2}+\mathbf{n}_{2} \times \mathbf{n}_{1} \sin \phi_{2}, \\
\mathbf{b}_{2} & =\left[\left(\mathbf{i} \cdot \mathbf{n}_{2}\right) \mathbf{n}_{1}+\left(\mathbf{i} \cdot \mathbf{n}_{1}\right) \mathbf{n}_{2}-\left(\mathbf{n}_{2} \cdot \mathbf{n}_{1}\right) \mathbf{i}\right] \sin \phi_{2}-\mathbf{n}_{2} \times \mathbf{n}_{1} \cos \phi_{2} .
\end{aligned}
$$

In principle, the parameters $\ell_{0}, \ell_{2}$ may be both positive or both negative (they must be nonzero for a regular curve, and cannot have opposite signs since the factor $\sqrt{\ell_{0} \ell_{2} z}$ in (36) should be real). However, if parameter values $\ell_{0}, \ell_{2}, \phi_{0}, \phi_{2}$ satisfy (53), then $-\ell_{0},-\ell_{2}, \phi_{0}+\pi, \phi_{2}+\pi$ also satisfy it, and define exactly the same curve. Hence, to avoid replication, we consider only solutions corresponding to positive $\ell_{0}$, $\ell_{2}$ values.

Now (53) is a vector equation in the three parameters $\ell_{0}, \ell_{2}, \phi_{1}$ in which $\mathbf{a}_{0}$, $\mathbf{b}_{0}, \mathbf{a}_{2}, \mathbf{b}_{2}, \mathbf{z}$ are known fixed vectors, and $z$ is a known constant. Taking its dot product with $\mathbf{i}, \mathbf{j}, \mathbf{k}$ gives the three scalar equations

$$
\begin{aligned}
\sqrt{\ell_{0} \ell_{2} z} \mathbf{i} \cdot\left[\left(\ell_{0} \mathbf{a}_{0}+\ell_{2} \mathbf{a}_{2}\right) \cos \phi_{1}\right. & \left.+\left(\ell_{0} \mathbf{b}_{0}+\ell_{2} \mathbf{b}_{2}\right) \sin \phi_{1}\right] \\
& +\ell_{0}^{2} \lambda_{i}+\ell_{2}^{2} \lambda_{f}+\ell_{0} \ell_{2} \mathbf{i} \cdot \mathbf{z}=5 L, \\
\sqrt{\ell_{0} \ell_{2} z} \mathbf{j} \cdot\left[\left(\ell_{0} \mathbf{a}_{0}+\ell_{2} \mathbf{a}_{2}\right) \cos \phi_{1}\right. & \left.+\left(\ell_{0} \mathbf{b}_{0}+\ell_{2} \mathbf{b}_{2}\right) \sin \phi_{1}\right] \\
& +\ell_{0}^{2} \mu_{i}+\ell_{2}^{2} \mu_{f}+\ell_{0} \ell_{2} \mathbf{j} \cdot \mathbf{z}=0, \\
\sqrt{\ell_{0} \ell_{2} z} \mathbf{k} \cdot\left[\left(\ell_{0} \mathbf{a}_{0}+\ell_{2} \mathbf{a}_{2}\right) \cos \phi_{1}\right. & \left.+\left(\ell_{0} \mathbf{b}_{0}+\ell_{2} \mathbf{b}_{2}\right) \sin \phi_{1}\right] \\
& +\ell_{0}^{2} \nu_{i}+\ell_{2}^{2} \nu_{f}+\ell_{0} \ell_{2} \mathbf{k} \cdot \mathbf{z}=0 .
\end{aligned}
$$

On dividing equations (56) and (57) by $\ell_{0}^{2}$, it is apparent that they depend only on the ratio

$$
\lambda=\frac{\ell_{2}}{\ell_{0}},
$$

and not on $\ell_{0}$ and $\ell_{2}$ individually. Furthermore, $\phi_{1}$ can be easily eliminated between these equations, to obtain a polynomial equation

$$
G(\lambda)=\sum_{k=0}^{6} g_{k} \lambda^{k}=0
$$

of degree 6 in $\lambda$ alone. This is accomplished by observing that (56) and (57) can be solved for $\cos \phi_{1}$ and $\sin \phi_{1}$ to obtain

$$
\cos \phi_{1}=\frac{(\mathbf{b} \times \mathbf{c}) \cdot \mathbf{i}}{(\mathbf{a} \times \mathbf{b}) \cdot \mathbf{i}} \quad \text { and } \quad \sin \phi_{1}=-\frac{(\mathbf{a} \times \mathbf{c}) \cdot \mathbf{i}}{(\mathbf{a} \times \mathbf{b}) \cdot \mathbf{i}},
$$

where we define

$$
\mathbf{a}=\sqrt{\lambda z}\left(\mathbf{a}_{0}+\lambda \mathbf{a}_{2}\right), \quad \mathbf{b}=\sqrt{\lambda z}\left(\mathbf{b}_{0}+\lambda \mathbf{b}_{2}\right), \quad \mathbf{c}=\mathbf{t}_{i}+\lambda^{2} \mathbf{t}_{f}+\lambda \mathbf{z} .
$$


Now expressions (60) imply that

$$
[(\mathbf{a} \times \mathbf{c}) \cdot \mathbf{i}]^{2}+[(\mathbf{b} \times \mathbf{c}) \cdot \mathbf{i}]^{2}=[(\mathbf{a} \times \mathbf{b}) \cdot \mathbf{i}]^{2},
$$

and on expanding and collecting like powers of $\lambda$, we obtain (59). Since the coefficients $g_{0}, \ldots, g_{6}$ are rather cumbersome, we defer them to Appendix 2.

To compute the positive real roots of (59), we observe that by introducing the change of variables specified by

$$
\lambda=\frac{\rho}{1-\rho},
$$

and setting $g_{k}=\tilde{g}_{k}\left(\begin{array}{l}6 \\ k\end{array}\right)$, this equation becomes

$$
\frac{1}{(1-\rho)^{6}} \sum_{k=0}^{6} \tilde{g}_{k}\left(\begin{array}{l}
6 \\
k
\end{array}\right)(1-\rho)^{6-k} \rho^{k}=0 .
$$

Then the positive real roots of (59) correspond to the roots $\rho \in[0,1)$ of this expression, which can be computed in a numerically stable manner [15] using standard algorithms for Bernstein-form polynomials.

Equation (59) must have at least one positive real root if an interpolant is to exist. For each positive root $\lambda$, two values of $\phi_{1}$ (that differ by $\pi$ ) are determined by equations (60). Corresponding $\lambda, \phi_{1}$ values obtained in this manner may be substituted into equation (55), with $\ell_{2}=\lambda \ell_{0}$, to obtain

$$
\ell_{0}^{2}=\frac{5 L}{\mathbf{i} \cdot\left[\sqrt{\lambda z}\left(\left(\mathbf{a}_{0}+\lambda \mathbf{a}_{2}\right) \cos \phi_{1}+\left(\mathbf{b}_{0}+\lambda \mathbf{b}_{2}\right) \sin \phi_{1}\right)+\mathbf{t}_{i}+\lambda^{2} \mathbf{t}_{f}+\lambda \mathbf{z}\right]} .
$$

For a real nonzero $\ell_{0}$ value, the expression on the right must be positive. If this requirement is met 7 we have $\ell_{2}=\lambda \ell_{0}$.

Remark 2. When the four possible $\left(\phi_{0}, \phi_{2}\right)$ pairs (50) are used in the above procedure, one finds that the first two pairs and last two pairs yield the same positive roots of (59), and for each root $\lambda$ the same $\ell_{0}, \ell_{2}$ values but $\phi_{1}$ values that differ by $\pi$. Now from (4), (6), (24) and (36) one can see that the triples $\left(\phi_{0}, \phi_{1}, \phi_{2}\right)$ and $\left(\phi_{0}+\pi, \phi_{1}+\pi, \phi_{2}+\pi\right)$ generate the same hodograph $\mathbf{r}^{\prime}(\xi)$. Hence, the solution yields only two effectively distinct $\left(\phi_{0}, \phi_{1}, \phi_{2}\right)$ triples.

Once the values of $\ell_{0}, \ell_{2}, \phi_{0}, \phi_{1}, \phi_{2}$ are determined as described above, the quaternion coefficients (24) and (36) are completely determined, and the construction of the quintic RRMF motion interpolant is complete. Setting $\mathcal{A}_{k}=$ $u_{k}+v_{k} \mathbf{i}+p_{k} \mathbf{j}+q_{k} \mathbf{k}$, the Hopf map coefficients are

$$
\boldsymbol{\alpha}_{k}=u_{k}+\mathrm{i} v_{k} \quad \text { and } \quad \boldsymbol{\beta}_{k}=q_{k}+\mathrm{i} p_{k}
$$

for $k=0,1,2$. The coefficients (40) can then be computed, and by identifying (17) with (38), the rational rotation-minimizing frame for the curve may be obtained from expressions (19), where $\mathcal{B}(\xi)$ is defined by (18).

\footnotetext{
${ }^{7}$ Note that the existence of solutions is independent of $L=|\Delta \mathbf{p}|$; this reflects the fact that the RMF is essentially unchanged under a uniform scaling.
} 
In the present context, the RMF vectors $(\mathbf{t}(\xi), \mathbf{u}(\xi), \mathbf{v}(\xi))$ defined by (19) are degree 8 rational functions in the curve parameter, since $\mathcal{A}(\xi)$ and $\mathcal{W}(\xi)$ in (18) are quadratic quaternion polynomials. By construction, this rational RMF satisfies the end frame interpolation conditions (11)-(2).

Remark 3. In cases where the polynomial (59) has no positive real roots, or the right-hand expression in (63) is negative - and thus no RRMF quintic motion interpolants exist - it may be possible to construct interpolants of higher degree by multiplying the hodograph (4) with a scalar polynomial $h(\xi)$ to introduce new free parameters. This does not change the RRMF condition or interpolation of the end frames, as described in Sections 3.1 3.3, it only alters the end-point interpolation. Experiments with a linear $h(\xi)$ reveal that interpolants can be obtained in cases where none exist with nominal RRMF quintics. A detailed analysis of this method, and of existence conditions for nominal RRMF quintic interpolants, is deferred to a future study.

3.5. Summary of algorithm. Since the above procedure is rather involved, we summarize it as follows.

Algorithm

input: initial/final points $\mathbf{p}_{i}, \mathbf{p}_{f}$ and frames $\left(\mathbf{t}_{i}, \mathbf{u}_{i}, \mathbf{v}_{i}\right),\left(\mathbf{t}_{f}, \mathbf{u}_{f}, \mathbf{v}_{f}\right)$

1. compute $\mathbf{n}_{0}, \mathbf{n}_{2}$ and $\gamma, \delta$ from (25) and (33), and the vectors $\mathbf{j}_{0}, \mathbf{j}_{2}, \mathbf{k}_{0}, \mathbf{k}_{2}$ from (42);

2. determine the values of $\phi_{0}$ from equations (44);

3 . from (49) identify the correct $\beta$ values, satisfying conditions (71)-(72) in Appendix 1, and set $\phi_{2}=\beta+\phi_{0}$;

4. compute the quantities $\mathbf{z}, z, \mathbf{n}_{1}, \mathbf{a}_{0}, \mathbf{a}_{2}, \mathbf{b}_{0}, \mathbf{b}_{2}$ defined by (30), (35), (37), and (54);

5. compute the coefficients (173) of the polynomial (59), and identify its positive real roots $\lambda$;

6. for each $\lambda$, compute the corresponding $\phi_{1}$ and $\ell_{0}$ values from (60) and (63), and set $\ell_{2}=\lambda \ell_{0}$;

7. determine the quaternion coefficients $\mathcal{A}_{0}, \mathcal{A}_{1}, \mathcal{A}_{2}$ from (24) and (36);

8. compute the Bézier control points $\mathbf{p}_{0}, \ldots, \mathbf{p}_{5}$ from (7);

9. using (64) and (40), determine the rational RMF $(\mathbf{t}(\xi), \mathbf{u}(\xi), \mathbf{v}(\xi))$ from (18) and (19), where (17) is identified with (38).

output: quintic RRMF curve $\mathbf{r}(\xi)$ with associated frame $(\mathbf{t}(\xi), \mathbf{u}(\xi), \mathbf{v}(\xi))$ that interpolates the prescribed data.

\section{Computed examples}

The following examples illustrate the procedure. An end-point displacement of the form $\Delta \mathbf{p}=\mathbf{p}_{f}-\mathbf{p}_{i}=(L, 0,0)$ is assumed in these examples, and for brevity we only list values of the $\left(\phi_{0}, \phi_{1}, \phi_{2}\right)$ variables that generate distinct RRMF quintic interpolants; see Remark 2 
Example 1. With $L=1$, consider the initial and final frame orientations

$$
\begin{aligned}
\mathbf{t}_{i} & =(0.707107,0.707107,0.000000), \\
\mathbf{u}_{i} & =(0.000000,0.000000,-1.000000), \\
\mathbf{v}_{i} & =(-0.707107,0.707107,0.000000), \\
\mathbf{t}_{f} & =(0.804738,-0.310617,0.505879), \\
\mathbf{u}_{f} & =(0.310617,-0.505879,-0.804738), \\
\mathbf{v}_{f} & =(0.505879,0.804738,-0.310617)
\end{aligned}
$$

In this case, the values (33) are

$$
\gamma=-0.101898 \quad \text { and } \quad \delta=0.815055,
$$

and from (44) and (49) with $\beta=\phi_{2}-\phi_{0}$ we obtain

$$
\phi_{0}=0.785398, \quad \phi_{2}=-0.345273, \quad \beta=-1.130672 .
$$

Equation (59) then has the two positive real roots

$$
\lambda=0.950478 \quad \text { and } \quad \lambda=1.437231 .
$$

For the first solution, we obtain from (60) and (63)

$$
\phi_{1}=1.146778, \quad l_{0}=1.388849, \quad l_{2}=1.320071,
$$

and hence the coefficients of the quaternion polynomial (6) and the complex polynomial (38), needed to construct the control points (7) and the rational RMF defined by (15) -(16), are given by

$$
\begin{gathered}
\mathcal{A}_{0}=-0.907309+0.907309 \mathbf{i}+0.375820 \mathbf{j}-0.375820 \mathbf{k}, \\
\mathcal{A}_{1}=-0.922515+0.416424 \mathbf{i}-0.346969 \mathbf{j}-0.025422 \mathbf{k}, \\
\mathcal{A}_{2}=0.424413+1.179970 \mathbf{i}-0.322053 \mathbf{j}+0.257706 \mathbf{k}, \\
\mathbf{w}_{0}=1, \quad \mathbf{w}_{1}=0.567156+0.310609 \mathbf{i}, \quad \mathbf{w}_{2}=0.593849-0.742127 \mathbf{i} .
\end{gathered}
$$

Satisfaction of the RRMF constraint (9) is verified by observing that

$$
\mathcal{A}_{1} \mathbf{i} \mathcal{A}_{1}^{*}=\operatorname{vect}\left(\mathcal{A}_{2} \mathbf{i} \mathcal{A}_{0}^{*}\right)=(0.903409,-0.242068,-0.661341) \text {. }
$$

For the second solution, we have

$$
\phi_{1}=-0.557987, \quad l_{0}=1.057830, \quad l_{2}=1.520346,
$$

and the coefficients of $\mathcal{A}(\xi)$ and $\mathbf{w}(\xi)$ are

$$
\begin{aligned}
& \mathcal{A}_{0}=-0.691061+0.691061 \mathbf{i}+0.286247 \mathbf{j}-0.286247 \mathbf{k}, \\
& \mathcal{A}_{1}= 0.501934+0.804189 \mathbf{i}+0.067003 \mathbf{j}-0.318878 \mathbf{k}, \\
& \mathcal{A}_{2}= 0.488803+1.358990 \mathbf{i}-0.370913 \mathbf{j}+0.296804 \mathbf{k}, \\
& \mathbf{w}_{0}=1, \quad \mathbf{w}_{1}=0.285373-0.742188 \mathbf{i}, \quad \mathbf{w}_{2}=0.897967-1.122180 \mathbf{i} .
\end{aligned}
$$

In this case we have

$$
\mathcal{A}_{1} \mathbf{i} \mathcal{A}_{1}^{*}=\operatorname{vect}\left(\mathcal{A}_{2} \mathbf{i} \mathcal{A}_{0}^{*}\right)=(0.792484,-0.212345,-0.580139) .
$$

Figure 2 shows the two RRMF quintic interpolants to the specified data, together with sampled positions of a rectangular parallelepiped whose center of mass traverses the curves at constant speed, and whose spatial orientation is specified by the rational RMF along them (the shortest parallelepiped side is aligned with the 

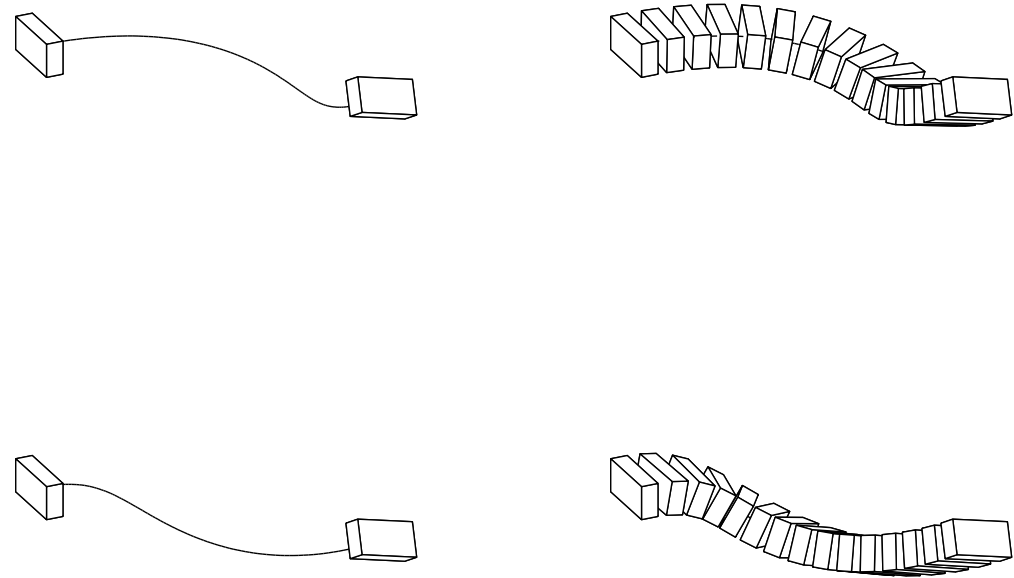

Figure 2. The two RRMF quintic interpolants of Example 1, for $\lambda=0.950478$ (upper) and 1.437231 (lower). Also shown (right) is the spatial motion of a rectangular parallelepiped along these paths, whose orientation is specified by their rational rotationminimizing frames. In both cases, the initial and final orientations agree with the prescribed Hermite data (left).

curve tangent $\mathbf{t}$, while the intermediate and longest sides are aligned with the two normal-plane RMF vectors $\mathbf{u}$ and $\mathbf{v}$ ).

Figure 3 shows two RRMF quintic interpolants to exactly the same data, except that $L$ is increased to 2 . This case illustrates the fact that the existence of solutions is independent of $L$, and the variation of the rational RMF when only $L$ is changed corresponds to uniform scaling of its angular velocity $\boldsymbol{\omega}$.

Example 2. Taking $L=1$ again, consider the end frame orientations

$$
\begin{aligned}
\mathbf{t}_{i} & =(0.866025,0.447214,-0.223607), \\
\mathbf{u}_{i} & =(-0.223607,-0.053590,-0.973205), \\
\mathbf{v}_{i} & =(-0.447214,0.892820,0.053590), \\
\mathbf{t}_{f} & =(0.583333,-0.623773,-0.520220), \\
\mathbf{u}_{f} & =(-0.186887,0.520220,-0.833333), \\
\mathbf{v}_{f} & =(0.790440,0.583333,0.186887) .
\end{aligned}
$$

In this case, we obtain

$$
\gamma=0.108248 \quad \text { and } \quad \delta=0.812130,
$$



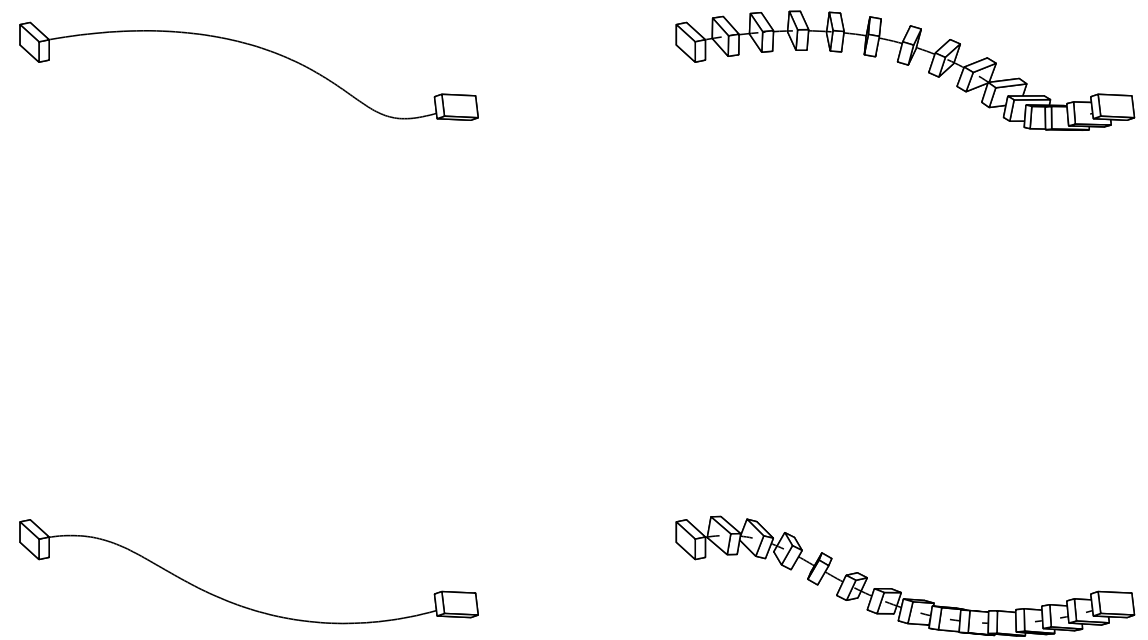

Figure 3. The RRMF quintic interpolants to the data of Example 1. except that $L$ is increased to 2 (note that the scale differs from that of Figure 2).

and

$$
\phi_{0}=0.785398, \quad \phi_{2}=1.910795, \quad \beta=1.125397 .
$$

Equation (59) has the two positive real solutions

$$
\lambda=0.557847 \quad \text { and } \quad \lambda=0.727110 .
$$

For the first solution, we have

$$
\phi_{1}=1.173752, \quad l_{0}=1.571261, \quad l_{2}=0.876524,
$$

and the coefficients of $\mathcal{A}(\xi)$ and $\mathbf{w}(\xi)$ are given by

$$
\begin{gathered}
\mathcal{A}_{0}=-1.073191+1.073191 \mathbf{i}+0.128601 \mathbf{j}-0.385803 \mathbf{k}, \\
\mathcal{A}_{1}=-0.807974+0.338794 \mathbf{i}+0.169303 \mathbf{j}+0.257659 \mathbf{k}, \\
\mathcal{A}_{2}=-0.735248-0.260083 \mathbf{i}-0.139110 \mathbf{j}+0.375113 \mathbf{k}, \\
\mathbf{w}_{0}=1, \quad \mathbf{w}_{1}=0.467045+0.164070 \mathbf{i}, \quad \mathbf{w}_{2}=0.349414+0.434860 \mathbf{i} .
\end{gathered}
$$

Checking the RRMF condition (9) then yields

$$
\mathcal{A}_{1} \mathbf{i} \mathcal{A}_{1}^{*}=\operatorname{vect}\left(\mathcal{A}_{2} \mathbf{i} \mathcal{A}_{0}^{*}\right)=(0.672552,-0.301645,0.448171) .
$$

For the second solution, we have

$$
\phi_{1}=2.043388, \quad l_{0}=1.531174, \quad l_{2}=1.113333,
$$


and hence

$$
\begin{aligned}
& \mathcal{A}_{0}=-1.045811+1.045811 \mathbf{i}+0.125320 \mathbf{j}-0.375960 \mathbf{k}, \\
& \mathcal{A}_{1}=-0.867897-0.443695 \mathbf{i}+0.340544 \mathbf{j}+0.041002 \mathbf{k}, \\
& \mathcal{A}_{2}=-0.933888-0.330350 \mathbf{i}-0.176693 \mathbf{j}+0.476456 \mathbf{k}, \\
& \mathbf{w}_{0}=1, \quad \mathbf{w}_{1}=0.200852+0.528263 \mathbf{i}, \quad \mathbf{w}_{2}=0.455434+0.566806 \mathbf{i},
\end{aligned}
$$

for the coefficients of $\mathcal{A}(\xi)$ and $\mathbf{w}(\xi)$. The RRMF condition (9) yields

$$
\mathcal{A}_{1} \text { i } \mathcal{A}_{1}^{*}=\operatorname{vect}\left(\mathcal{A}_{2} \text { i } \mathcal{A}_{0}^{*}\right)=(0.832459,-0.373366,0.554729) .
$$
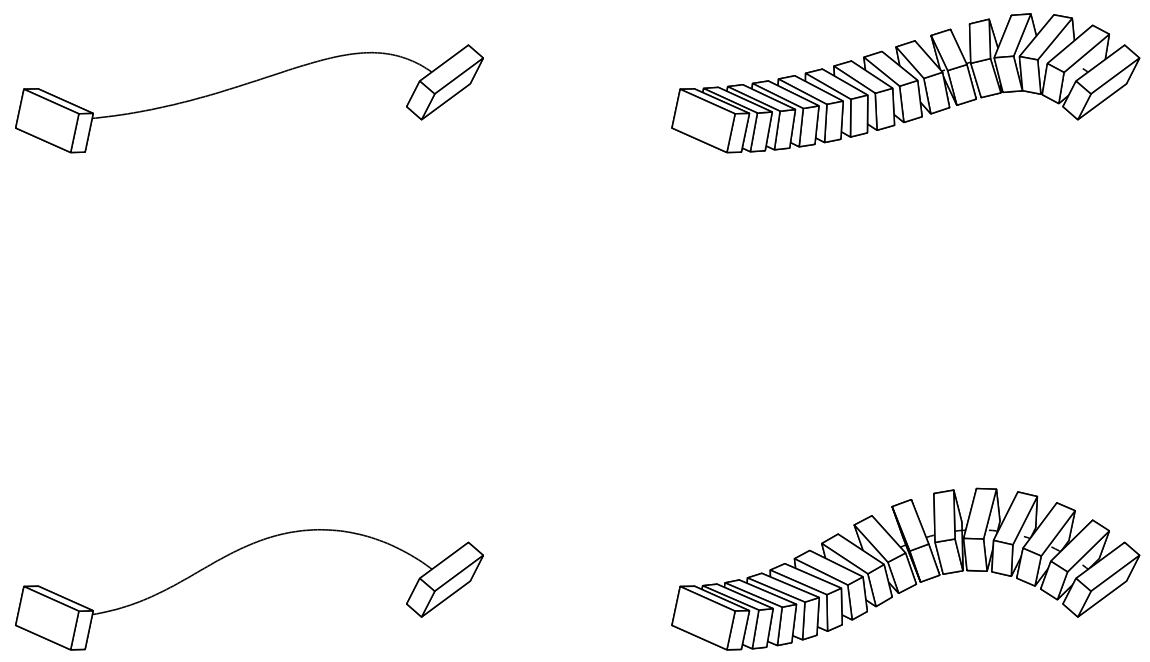

Figure 4. The two RRMF quintic interpolants of Example 2 for $\lambda=0.557847$ (upper) and 0.727110 (lower). Also shown (right) is the spatial motion of a rectangular parallelepiped along these paths, whose orientation is specified by their rational rotationminimizing frames. In both cases, the initial and final orientations agree with the prescribed Hermite data (left).

Figure 4 shows rational rotation-minimizing motions of a parallelepiped, analogous to those shown for Example 1 in Figure 2, in the case of Example 2. In Figure 5, we compare the motions generated along the second curve using the ERF and RMF to orient the parallelepiped along the path - the more "natural" motion afforded by the RMF is clearly apparent. 

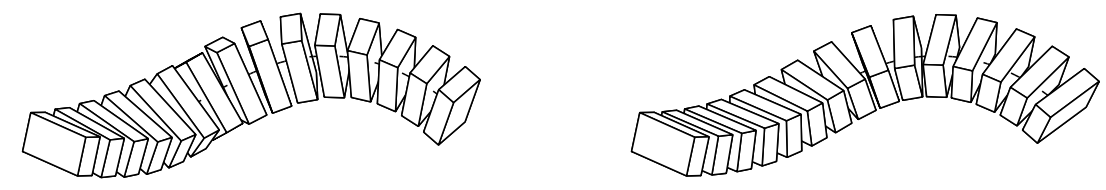

Figure 5. Comparison of orientations specified by the ERF (left) and RMF (right) along the RRMF quintic interpolant of Example 2 with $\lambda=0.727110$ (the orientations of the parallelepiped are coincident at the left end point).

Example 3. For $L=1$ and the initial and final frames

$$
\begin{aligned}
\mathbf{t}_{i} & =(0.500000,0.000000,0.866025), \\
\mathbf{u}_{i} & =(0.000000,1.000000,0.000000), \\
\mathbf{v}_{i} & =(-0.866025,0.00000,0.500000), \\
\mathbf{t}_{f} & =(0.500000,-0.707107,0.500000), \\
\mathbf{u}_{f} & =(0.707107,0.000000,-0.707107), \\
\mathbf{v}_{f} & =(0.500000,0.707107,0.500000),
\end{aligned}
$$

we have

$$
\gamma=-0.204124 \quad \text { and } \quad \delta=0.894338 \text {, }
$$

and the admissible values for $\left(\phi_{0}, \phi_{2}\right)$ are

$$
(1.570796,6.183905) \quad \text { and } \quad(1.570796,4.497600) \text {, }
$$

corresponding to $\beta=4.613109$ and $\beta=2.926804$, respectively. In both cases, however, equation (59) has no positive real roots, and therefore no RRMF quintic interpolants exist.

\section{Asymptotic AnALYsis}

We now use a Taylor series expansion to investigate the asymptotic existence of quintic RRMF curves interpolating given data of the form (11)-(2); i.e., we establish if, for asymptotic data, the polynomial (59) admits positive real roots, and the corresponding denominator on the right in (63) is positive.

The data for the asymptotic analysis are assumed to be sampled from an infinitesimal segment of a sufficiently smooth space curve $\mathbf{R}(s)$ parameterized by arc length $s$, and its associated $\operatorname{RMF}(\dot{\mathbf{R}}(s), \mathbf{u}(s), \mathbf{v}(s))$, which is fixed by imposing the initial condition

$$
\mathbf{u}(0)=\mathbf{w}, \quad \mathbf{v}(0)=\dot{\mathbf{R}}(0) \times \mathbf{w},
$$

where $\mathbf{w}$ is any unit vector orthogonal to $\dot{\mathbf{R}}(0)$. The arc-length derivatives of $\mathbf{R}(s)$ are denoted by $\dot{\mathbf{R}}(s), \ddot{\mathbf{R}}(s), \mathbf{R}^{(3)}(s), \mathbf{R}^{(4)}(s), \ldots$, etc. 
Although the use of arc-length parameterization somewhat simplifies the analysis, it was found expedient to use MAPLE in the symbolic computation of the 6thorder Taylor series expansion, required in the proof of the following result (the MAPLE worksheet is available from the authors upon request).

Proposition 3. Let $\mathbf{R}(s), s \in[0, \Delta s]$ be an arc-length parameterized regular space curve with $C^{7}$ continuity in a neighborhood of $s=0$, and nonvanishing curvature, derivative of curvature, and torsion at $s=0$. Then if the data of the motion problem in (1) -(2) are such that

$$
\begin{array}{ll}
\mathbf{p}_{i}=\mathbf{R}(0), & \left(\mathbf{t}_{i}, \mathbf{u}_{i}, \mathbf{v}_{i}\right)=(\dot{\mathbf{R}}(0), \mathbf{u}(0), \mathbf{v}(0)), \\
\mathbf{p}_{f}=\mathbf{R}(\Delta s), & \left(\mathbf{t}_{f}, \mathbf{u}_{f}, \mathbf{v}_{f}\right)=(\dot{\mathbf{R}}(\Delta s), \mathbf{u}(\Delta s), \mathbf{v}(\Delta s)),
\end{array}
$$

where $(\dot{\mathbf{R}}(s), \mathbf{u}(s), \mathbf{v}(s))$ is an RMF on $\mathbf{R}(s)$, the polynomial (59) has at least one positive real root and the corresponding value for the right-hand side of (63) is positive, when $\Delta s$ is sufficiently small.

Proof. The proof uses a 6 th-order Taylor expansion of $\mathbf{R}(s)$ about $s=0$. Without loss of generality, we may choose

$$
\mathbf{R}(0)=\mathbf{0}, \quad \dot{\mathbf{R}}(0)=\mathbf{i}, \quad \mathbf{u}(0)=\mathbf{w}=\mathbf{j}, \quad \mathbf{v}(0)=\mathbf{k}
$$

at $s=0$. As the arc length parameterization satisfies

$$
\dot{\mathbf{R}}(s) \cdot \ddot{\mathbf{R}}(s) \equiv 0,
$$

we may set

$$
\ddot{\mathbf{R}}(0)=b_{y} \mathbf{j}+b_{z} \mathbf{k} \quad \text { where } b_{y}^{2}+b_{z}^{2} \neq 0,
$$

since the curvature is assumed nonzero at $s=0$. Differentiating (65) then gives $\mathbf{i} \cdot \mathbf{R}^{(3)}(0)=-|\ddot{\mathbf{R}}(0)|^{2}=-\left(b_{y}^{2}+b_{z}^{2}\right)$. Hence, we may write

$$
\mathbf{R}^{(3)}(0)=-\left(b_{y}^{2}+b_{z}^{2}\right) \mathbf{i}+c_{y} \mathbf{j}+c_{z} \mathbf{k},
$$

where the values $c_{y}, c_{z}$ are such that

$$
b_{y} c_{y}+b_{z} c_{z} \neq 0 \quad \text { and } \quad b_{y} c_{z}-b_{z} c_{y} \neq 0
$$

because of the assumed nonzero curvature derivative and torsion at $s=0$. Successively differentiating (65) and evaluating at $s=0$, we may also write

$$
\begin{aligned}
& \mathbf{R}^{(4)}(0)=d_{x} \mathbf{i}+d_{y} \mathbf{j}+d_{z} \mathbf{k}, \\
& \mathbf{R}^{(5)}(0)=e_{x} \mathbf{i}+e_{y} \mathbf{j}+e_{z} \mathbf{k}, \\
& \mathbf{R}^{(6)}(0)=f_{x} \mathbf{i}+f_{y} \mathbf{j}+f_{z} \mathbf{k},
\end{aligned}
$$

where

$$
\begin{aligned}
d_{x} & =-3 \ddot{\mathbf{R}}(0) \cdot \mathbf{R}^{(3)}(0), \\
e_{x} & =-4 \ddot{\mathbf{R}}(0) \cdot \mathbf{R}^{(4)}(0)-3\left|\mathbf{R}^{(3)}(0)\right|^{2}, \\
f_{x} & =-5 \ddot{\mathbf{R}}(0) \cdot \mathbf{R}^{(5)}(0)-10 \mathbf{R}^{(3)}(0) \cdot \mathbf{R}^{(4)}(0) .
\end{aligned}
$$

In the following analysis, the scalar parameters $b_{y}, b_{z}, c_{y}, c_{z}, d_{y}, d_{z}, e_{y}, e_{z}, f_{y}, f_{z}$ introduced above are regarded as symbolic variables.

To obtain the necessary Taylor expansion of $\mathbf{u}(s)$ about $s=0$, we use the differential equation

$$
\dot{\mathbf{u}}=-(\mathbf{u} \cdot \ddot{\mathbf{R}}) \dot{\mathbf{R}}
$$


(expressed in terms of arc-length derivatives) that identifies $\mathbf{u}$ as a rotation-minimizing unit frame vector [21 in the normal plane of $\mathbf{R}(s)$. Successively differentiating this equation, we obtain expressions for $\dot{\mathbf{u}}(0), \ddot{\mathbf{u}}(0), \ldots, \mathbf{u}^{(5)}(0)$ in terms of the symbolic parameters $b_{y}, b_{z}, \ldots, f_{y}, f_{z}$ defined above.

Evaluating the Taylor expansions at $s=\Delta s$ gives expressions of the form

$$
\begin{aligned}
\mathbf{R}(\Delta s) & =\Delta s \mathbf{i}+\frac{\Delta s^{2}}{2} \ddot{\mathbf{R}}(0)+\cdots+\frac{\Delta s^{6}}{6 !} \mathbf{R}^{(6)}(0)+\mathrm{O}\left(\Delta s^{7}\right), \\
\dot{\mathbf{R}}(\Delta s) & =\mathbf{i}+\Delta s \ddot{\mathbf{R}}(0)+\cdots+\frac{\Delta s^{5}}{5 !} \mathbf{R}^{(6)}(0)+\mathrm{O}\left(\Delta s^{6}\right), \\
\mathbf{u}(\Delta s) & =\mathbf{j}+\Delta s \dot{\mathbf{u}}(0)+\cdots+\frac{\Delta s^{5}}{5 !} \mathbf{u}^{(5)}(0)+\mathrm{O}\left(\Delta s^{6}\right), \\
\mathbf{v}(\Delta s) & =\dot{\mathbf{R}}(\Delta s) \times \mathbf{u}(\Delta s) .
\end{aligned}
$$

By using these expansions, we can obtain an asymptotic expression for the polynomial (59) of the form

$$
G(\lambda)=\sum_{i=0}^{4} P_{i}(\lambda)(\lambda-1)^{4-i} \Delta s^{4+i}+O\left(\Delta s^{9}\right),
$$

where $P_{i}(\lambda), i=0, \ldots, 4$ are polynomials of degree $2+i$ such that $P_{i}(1) \neq 0$, with coefficients dependent on the parameters $b_{y}, b_{z}, \ldots, f_{y}, f_{z}$. Evaluating this expression at $\lambda=1+\zeta \Delta s$ then gives

$$
G(1+\zeta \Delta s)=p(\zeta) \Delta s^{8}+O\left(\Delta s^{9}\right),
$$

where $p(\zeta)=\sum_{i=0}^{4} P_{i}(1) \zeta^{4-i}$. Using MAPLE one can verify that, under the stated assumptions, this polynomial always has the value

$$
\hat{\zeta}=\frac{b_{y} c_{y}+b_{z} c_{z}}{3\left(b_{y}^{2}+b_{z}^{2}\right)}
$$

as a simple root. Thus, there exists a positive $\epsilon$ such that $p(\hat{\zeta}-\epsilon) p(\hat{\zeta}+\epsilon)<0$, which implies that $G(1+(\hat{\zeta}-\epsilon) \Delta s) G(1+(\hat{\zeta}+\epsilon) \Delta s)<0$ for a sufficiently small $\Delta s$ such that $1+(\hat{\zeta}-\epsilon) \Delta s>0$. Hence, we can conclude that there exists a positive real root $\hat{\lambda}$ of $G(\lambda)$, with odd multiplicity, on the interval $[1+(\hat{\zeta}-\epsilon) \Delta s, 1+(\hat{\zeta}+\epsilon) \Delta s]$. Assuming $\lambda=\hat{\lambda}=1+O(\Delta s)$, a further MAPLE computation allows us to verify that the Taylor expansion of the denominator on the right-hand side of (63) has a positive zeroorder term in $\Delta s$. Hence, the statement of the proposition is proved.

\section{Closure}

A method for computing exact rational rotation-minimizing motions of rigid bodies, that interpolate prescribed initial/final positions $\mathbf{p}_{i}$ and $\mathbf{p}_{f}$ and frame orientations $\left(\mathbf{t}_{i}, \mathbf{u}_{i}, \mathbf{v}_{i}\right)$ and $\left(\mathbf{t}_{f}, \mathbf{u}_{f}, \mathbf{v}_{f}\right)$, has been presented. Such motions are specified by a quintic RRMF curve $\mathbf{r}(\xi)$ and associated rational orthonormal frame $(\mathbf{t}(\xi), \mathbf{u}(\xi), \mathbf{v}(\xi))$ of degree 8 , and have the characteristic property that the frame angular velocity $\boldsymbol{\omega}(\xi)$ has zero component along the curve tangent $\mathbf{t}(\xi)=\mathbf{r}^{\prime}(\xi) /\left|\mathbf{r}^{\prime}(\xi)\right|$; i.e., $\mathbf{t}(\xi) \cdot \boldsymbol{\omega}(\xi) \equiv 0$ and the two normal-plane vectors $\mathbf{u}(\xi), \mathbf{v}(\xi)$ exhibit no instantaneous rotation about $\mathbf{t}(\xi)$.

The method is expected to be useful for diverse applications in which the coordinated translational and orientational motion of a body is of interest, such as 
computer animation, robot end-effector manipulation, swept surface constructions in computer-aided geometric design, and spatial path planning for computer control of multi-axis manufacturing/inspection machines.

Two key components of the method - satisfaction of the constraint that identifies the RRMF curves among all spatial $\mathrm{PH}$ quintics, and interpolation of the end frames — were shown to admit unique closed-form solutions. The remaining component — interpolation of the end-point displacement — can be reduced to finding the positive real roots of a degree 6 polynomial. Since the coefficients of this polynomial have a complicated nonlinear dependence on the initial data, no attempt has been made at present to identify general conditions for the existence of solutions (although it has been verified in the case of data sampled asymptotically from smooth analytic curves).

The identification of general constraints on the initial data $\mathbf{p}_{i},\left(\mathbf{t}_{i}, \mathbf{u}_{i}, \mathbf{v}_{i}\right)$ and $\mathbf{p}_{f},\left(\mathbf{t}_{f}, \mathbf{u}_{f}, \mathbf{v}_{f}\right)$ that are sufficient and necessary for existence of solutions, and also modifications to the displacement interpolation problem that permit solutions in cases where none exist, will be addressed in future studies.

Appendix 1. Derivation of equation (48). If a quaternion $\mathcal{A}$ is given in terms of a scalar $\ell$, a vector $\mathbf{v}=v_{x} \mathbf{i}+v_{y} \mathbf{j}+v_{z} \mathbf{k}$, and an angular parameter $\phi$, in the form

$$
\mathcal{A}=\ell \frac{v \mathbf{i}+\mathbf{v}}{|v \mathbf{i}+\mathbf{v}|} \exp (\phi \mathbf{i})
$$

it can be expressed as $\mathcal{A}=\boldsymbol{\alpha}+\mathbf{k} \boldsymbol{\beta}$, in terms of the complex numbers

$$
\boldsymbol{\alpha}=\frac{\ell\left(v+v_{x}\right) \mathrm{i}}{|v \mathbf{i}+\mathbf{v}|} \mathrm{e}^{\mathrm{i} \phi} \quad \text { and } \quad \boldsymbol{\beta}=\frac{\ell\left(v_{z}+\mathrm{i} v_{y}\right)}{|v \mathbf{i}+\mathbf{v}|} \mathrm{e}^{\mathrm{i} \phi},
$$

when we identify the imaginary unit $\mathrm{i}$ with the quaternion basis element $\mathbf{i}$.

Thus, writing $\mathbf{z}=z(l, m, n)$ where $l^{2}+m^{2}+n^{2}=1$, the complex numbers $\boldsymbol{\alpha}_{i}, \boldsymbol{\beta}_{i}$ for $i=0,1,2$ that specify the Hopf map form (5) corresponding to the quaternion coefficients (24) and (36) can be written as

$$
\begin{array}{ccc}
\boldsymbol{\alpha}_{0}=\frac{\ell_{0}\left(1+\lambda_{i}\right) \mathrm{i}}{\sqrt{2\left(1+\lambda_{i}\right)}} \mathrm{e}^{\mathrm{i} \phi_{0}}, & \boldsymbol{\beta}_{0}=\frac{\ell_{0}\left(\nu_{i}+\mathrm{i} \mu_{i}\right)}{\sqrt{2\left(1+\lambda_{i}\right)}} \mathrm{e}^{\mathrm{i} \phi_{0}}, \\
\boldsymbol{\alpha}_{1}=\frac{\sqrt{\ell_{0} \ell_{2} z}(1+l) \mathrm{i}}{\sqrt{2(1+l)}} \mathrm{e}^{\mathrm{i} \phi_{1}}, & \boldsymbol{\beta}_{1}=\frac{\sqrt{\ell_{0} \ell_{2} z}(n+\mathrm{i} m)}{\sqrt{2(1+l)}} \mathrm{e}^{\mathrm{i} \phi_{1}}, \\
\boldsymbol{\alpha}_{2}=\frac{\ell_{2}\left(1+\lambda_{f}\right) \mathrm{i}}{\sqrt{2\left(1+\lambda_{f}\right)}} \mathrm{e}^{\mathrm{i} \phi_{2}}, & \boldsymbol{\beta}_{2}=\frac{\ell_{2}\left(\nu_{f}+\mathrm{i} \mu_{f}\right)}{\sqrt{2\left(1+\lambda_{f}\right)}} \mathrm{e}^{\mathrm{i} \phi_{2}} .
\end{array}
$$

To reduce relation (47) to the quadratic equation (48) in $\tan \beta$, we observe that the numerator on the right in (47) can be expressed as

$$
\begin{aligned}
\left(\overline{\boldsymbol{\alpha}}_{1} \boldsymbol{\alpha}_{2}+\overline{\boldsymbol{\beta}}_{1} \boldsymbol{\beta}_{2}\right)\left(\overline{\boldsymbol{\alpha}}_{0} \boldsymbol{\alpha}_{1}+\overline{\boldsymbol{\beta}}_{0} \boldsymbol{\beta}_{1}\right) & =\left(\left|\boldsymbol{\alpha}_{1}\right|^{2}+\left|\boldsymbol{\beta}_{1}\right|^{2}\right)\left(\overline{\boldsymbol{\alpha}}_{0} \boldsymbol{\alpha}_{2}+\overline{\boldsymbol{\beta}}_{0} \boldsymbol{\beta}_{2}\right) \\
& +\left(\overline{\boldsymbol{\alpha}}_{0} \overline{\boldsymbol{\beta}}_{1}-\overline{\boldsymbol{\alpha}}_{1} \overline{\boldsymbol{\beta}}_{0}\right)\left(\boldsymbol{\alpha}_{1} \boldsymbol{\beta}_{2}-\boldsymbol{\alpha}_{2} \boldsymbol{\beta}_{1}\right) .
\end{aligned}
$$

Now from the expressions (67)-69) for $\boldsymbol{\alpha}_{i}, \boldsymbol{\beta}_{i}$ we obtain

$$
\begin{gathered}
\left|\boldsymbol{\alpha}_{1}\right|^{2}+\left|\boldsymbol{\beta}_{1}\right|^{2}=\ell_{0} \ell_{2} z \\
\overline{\boldsymbol{\alpha}}_{0} \boldsymbol{\alpha}_{2}+\overline{\boldsymbol{\beta}}_{0} \boldsymbol{\beta}_{2}=\frac{\ell_{0} \ell_{2} \mathrm{e}^{\mathrm{i}\left(\phi_{2}-\phi_{0}\right)}\left[\left(1+\lambda_{i}\right)\left(1+\lambda_{f}\right)+\left(\mu_{i}+\mathrm{i} \nu_{i}\right)\left(\mu_{f}-\mathrm{i} \nu_{f}\right)\right]}{2 \sqrt{\left(1+\lambda_{i}\right)\left(1+\lambda_{f}\right)}},
\end{gathered}
$$




$$
\begin{aligned}
\overline{\boldsymbol{\alpha}}_{0} \overline{\boldsymbol{\beta}}_{1}-\overline{\boldsymbol{\alpha}}_{1} \overline{\boldsymbol{\beta}}_{0}=\frac{\ell_{0} \sqrt{\ell_{0} \ell_{2} z} \mathrm{e}^{-\mathrm{i}\left(\phi_{0}+\phi_{1}\right)}\left[\left(\mu_{i}+\mathrm{i} \nu_{i}\right)(1+l)-\left(1+\lambda_{i}\right)(m+\mathrm{i} n)\right]}{2 \sqrt{\left(1+\lambda_{i}\right)(1+l)}}, \\
\boldsymbol{\alpha}_{1} \boldsymbol{\beta}_{2}-\boldsymbol{\alpha}_{2} \boldsymbol{\beta}_{1}=\frac{\ell_{2} \sqrt{\ell_{0} \ell_{2} z} \mathrm{e}^{\mathrm{i}\left(\phi_{1}+\phi_{2}\right)}\left[\left(1+\lambda_{f}\right)(m-\mathrm{i} n)-\left(\mu_{f}-\mathrm{i} \nu_{f}\right)(1+l)\right]}{2 \sqrt{\left(1+\lambda_{f}\right)(1+l)}} .
\end{aligned}
$$

Substituting these into (70) and using $l^{2}+m^{2}+n^{2}=1$ then gives

$$
\left(\overline{\boldsymbol{\alpha}}_{1} \boldsymbol{\alpha}_{2}+\overline{\boldsymbol{\beta}}_{1} \boldsymbol{\beta}_{2}\right)\left(\overline{\boldsymbol{\alpha}}_{0} \boldsymbol{\alpha}_{1}+\overline{\boldsymbol{\beta}}_{0} \boldsymbol{\beta}_{1}\right)=\frac{\ell_{0}^{2} \ell_{2}^{2} \mathrm{e}^{\mathrm{i}\left(\phi_{2}-\phi_{0}\right)}\left(\mathbf{c}_{0} z+\mathbf{c}_{1} l z+\mathbf{c}_{2} m z+\mathbf{c}_{3} n z\right)}{4 \sqrt{\left(1+\lambda_{i}\right)\left(1+\lambda_{f}\right)}},
$$

where we set

$$
\begin{aligned}
& \mathbf{c}_{0}=\left(1+\lambda_{i}\right)\left(1+\lambda_{f}\right)+\left(\mu_{i}+\mathrm{i} \nu_{i}\right)\left(\mu_{f}-\mathrm{i} \nu_{f}\right), \\
& \mathbf{c}_{1}=\left(1+\lambda_{i}\right)\left(1+\lambda_{f}\right)-\left(\mu_{i}+\mathrm{i} \nu_{i}\right)\left(\mu_{f}-\mathrm{i} \nu_{f}\right), \\
& \mathbf{c}_{2}=\left(1+\lambda_{i}\right)\left(\mu_{f}-\mathrm{i} \nu_{f}\right)+\left(1+\lambda_{f}\right)\left(\mu_{i}+\mathrm{i} \nu_{i}\right), \\
& \mathbf{c}_{3}=\left(1+\lambda_{i}\right)\left(\mu_{f}-\mathrm{i} \nu_{f}\right)-\left(1+\lambda_{f}\right)\left(\mu_{i}+\mathrm{i} \nu_{i}\right) .
\end{aligned}
$$

Hence, the relation (47) becomes

$$
\mathrm{e}^{\mathrm{i}\left(\phi_{2}-\eta\right)}=\mathrm{e}^{\mathrm{i}\left(\phi_{2}-\phi_{0}\right)} \frac{\mathbf{c}_{0} z+\mathbf{c}_{1} l z+\mathbf{c}_{2} m z+\mathbf{c}_{3} n z}{\left|\mathbf{c}_{0} z+\mathbf{c}_{1} l z+\mathbf{c}_{2} m z+\mathbf{c}_{3} n z\right|} .
$$

Multiplying by $\mathrm{e}^{-\mathrm{i}\left(\phi_{2}-\phi_{0}\right)}$ and setting $(l z, m z, n z)=(\mathbf{i} \cdot \mathbf{z}, \mathbf{j} \cdot \mathbf{z}, \mathbf{k} \cdot \mathbf{z})$ where $\mathbf{z}$ is given by (30), a straightforward but laborious calculation yields

$$
\mathrm{e}^{\mathrm{i}\left(\phi_{0}-\eta\right)}=\frac{\boldsymbol{\zeta}_{0} z(\beta)-\boldsymbol{\zeta}_{1} \cos \beta-\boldsymbol{\zeta}_{2} \sin \beta}{\left|\boldsymbol{\zeta}_{0} z(\beta)-\boldsymbol{\zeta}_{1} \cos \beta-\boldsymbol{\zeta}_{2} \sin \beta\right|},
$$

where $\beta=\phi_{2}-\phi_{0}$ and $\boldsymbol{\zeta}_{0}, \boldsymbol{\zeta}_{1}, \boldsymbol{\zeta}_{2}$ are given in terms of the quantities (33) as

$$
\boldsymbol{\zeta}_{0}=\delta+\mathrm{i} \gamma, \quad \boldsymbol{\zeta}_{1}=\gamma^{2}-1-\mathrm{i} \gamma \delta, \quad \boldsymbol{\zeta}_{2}=\gamma \delta+\mathrm{i}\left(1-\delta^{2}\right) .
$$

Thus, writing $t=\tan \left(\phi_{0}-\eta\right)$, equation (71) implies that

$$
t=\frac{\gamma z(\beta)+\gamma \delta \cos \beta-\left(1-\delta^{2}\right) \sin \beta}{\delta z(\beta)-\left(\gamma^{2}-1\right) \cos \beta-\gamma \delta \sin \beta}
$$

or

$$
(\gamma-\delta t) z(\beta)=\left[\left(1-\gamma^{2}\right) t-\gamma \delta\right] \cos \beta+\left[1-\gamma \delta t-\delta^{2}\right] \sin \beta
$$

On squaring both sides of this equation and using (35), it yields the quadratic equation (48). The roots of the latter should be checked by substituting them into (71) and (72), since equating tangents and squaring introduces spurious roots that do not satisfy these conditions.

Appendix 2. Coefficients of equation (59). Equation (59) is obtained by expanding the relation (61) and collecting like powers of $\lambda$. This gives the coefficients $g_{0}, \ldots, g_{6}$ in terms of the end tangents $\mathbf{t}_{i}, \mathbf{t}_{f}$ and the quantities (30), (32), (54) as follows. Writing

$$
d_{0}=\left(\mathbf{a}_{0} \times \mathbf{b}_{0}\right) \cdot \mathbf{i}, \quad d_{1}=\left(\mathbf{a}_{0} \times \mathbf{b}_{2}+\mathbf{a}_{2} \times \mathbf{b}_{0}\right) \cdot \mathbf{i}, \quad d_{2}=\left(\mathbf{a}_{2} \times \mathbf{b}_{2}\right) \cdot \mathbf{i}
$$

and

$$
\begin{array}{lll}
e_{0}=\left(\mathbf{a}_{0} \times \mathbf{t}_{i}\right) \cdot \mathbf{i}, & f_{0}=\left(\mathbf{b}_{0} \times \mathbf{t}_{i}\right) \cdot \mathbf{i}, \\
e_{1}=\left(\mathbf{a}_{0} \times \mathbf{z}+\mathbf{a}_{2} \times \mathbf{t}_{i}\right) \cdot \mathbf{i}, & & f_{1}=\left(\mathbf{b}_{0} \times \mathbf{z}+\mathbf{b}_{2} \times \mathbf{t}_{i}\right) \cdot \mathbf{i}, \\
e_{2}=\left(\mathbf{a}_{0} \times \mathbf{t}_{f}+\mathbf{a}_{2} \times \mathbf{z}\right) \cdot \mathbf{i}, & f_{2}=\left(\mathbf{b}_{0} \times \mathbf{t}_{f}+\mathbf{b}_{2} \times \mathbf{z}\right) \cdot \mathbf{i}, \\
e_{3}=\left(\mathbf{a}_{2} \times \mathbf{t}_{f}\right) \cdot \mathbf{i}, & f_{3}=\left(\mathbf{b}_{2} \times \mathbf{t}_{f}\right) \cdot \mathbf{i},
\end{array}
$$


we obtain

$$
\begin{aligned}
& g_{0}=e_{0}^{2}+f_{0}^{2}, \\
& g_{1}=2\left(e_{0} e_{1}+f_{0} f_{1}\right)-z d_{0}^{2}, \\
& g_{2}=e_{1}^{2}+f_{1}^{2}+2\left(e_{0} e_{2}+f_{0} f_{2}\right)-2 z d_{0} d_{1}, \\
& g_{3}=2\left(e_{1} e_{2}+e_{0} e_{3}+f_{1} f_{2}+f_{0} f_{3}\right)-z\left(d_{1}^{2}+2 d_{0} d_{2}\right), \\
& g_{4}=e_{2}^{2}+f_{2}^{2}+2\left(e_{1} e_{3}+f_{1} f_{3}\right)-2 z d_{1} d_{2}, \\
& g_{5}=2\left(e_{2} e_{3}+f_{2} f_{3}\right)-z d_{2}^{2}, \\
& g_{6}=e_{3}^{2}+f_{3}^{2} .
\end{aligned}
$$

\section{REFERENCES}

[1] G. Albrecht and R. T. Farouki (1996), Construction of $C^{2}$ Pythagorean-hodograph interpolating splines by the homotopy method, Adv. Comp. Math. 5, 417-442. MR1414289 (97k:65033)

[2] H. I. Choi and C. Y. Han (2002), Euler-Rodrigues frames on spatial Pythagorean-hodograph curves, Comput. Aided Geom. Design 19, 603-620. MR.1937124 (2003i:53002)

[3] H. I. Choi, D. S. Lee, and H. P. Moon (2002), Clifford algebra, spin representation, and rational parameterization of curves and surfaces, Adv. Comp. Math. 17, 5-48. MR1902534 (2003c:53003)

[4] G. Farin (1997), Curves and Surfaces for Computer Aided Geometric Design, 4th edition, Academic Press, San Diego. MR1412572 (97e:65022)

[5] R. T. Farouki (2008), Pythagorean-Hodograph Curves: Algebra and Geometry Inseparable, Springer, Berlin. MR2365013 (2008k:65027)

[6] R. T. Farouki (2010), Quaternion and Hopf map characterizations for the existence of rational rotation-minimizing frames on quintic space curves, Adv. Comp. Math. 33, 331-348. MR2718102

[7] R. T. Farouki, M. al-Kandari, and T. Sakkalis (2002), Structural invariance of spatial Pythagorean hodographs, Comput. Aided Geom. Design 19, 395-407. MR1917337 (2003g:65019)

[8] R. T. Farouki, M. al-Kandari, and T. Sakkalis (2002), Hermite interpolation by rotation-invariant spatial Pythagorean-hodograph curves, Adv. Comp. Math. 17, 369-383. MR1916985 (2003e:65011)

[9] R. T. Farouki, C. Giannelli, C. Manni, and A. Sestini (2008), Identification of spatial PH quintic Hermite interpolants with near-optimal shape measures, Comput. Aided Geom. Design 25, 274-297. MR2408092 (2009c:65020)

[10] R. T. Farouki, C. Giannelli, C. Manni, and A. Sestini (2009), Quintic space curves with rational rotation-minimizing frames, Comput. Aided Geom. Design 26, 580-592. MR 2526013 (2010c:65023)

[11] R. T. Farouki, C. Giannelli, and A. Sestini (2010), Geometric design using space curves with rational rotation-minimizing frames, in (M. Daehlen et al., eds.), Lecture Notes in Computer Science Vol. 5862, pp. 194-208, Springer.

[12] R. T. Farouki and C. Y. Han (2003), Rational approximation schemes for rotation-minimizing frames on Pythagorean-hodograph curves, Comput. Aided Geom. Design 20, 435-454. MR2011551 (2004m:65025)

[13] R. T. Farouki, B. K. Kuspa, C. Manni, and A. Sestini (2001), Efficient solution of the complex quadratic tridiagonal system for $C^{2} \mathrm{PH}$ quintic splines, Numer. Algor. 27, 35-60. MR 1847983 (2002e:65074)

[14] R. T. Farouki and C. A. Neff (1995), Hermite interpolation by Pythagorean-hodograph quintics, Math. Comp. 64, 1589-1609. MR.1308452 (95m:65025)

[15] R. T. Farouki and V. T. Rajan (1987), On the numerical condition of polynomials in Bernstein form, Comput. Aided Geom. Design 4, 191-216. MR917780 (89a:65028)

[16] R. T. Farouki and T. Sakkalis (2010), Rational rotation-minimizing frames on polynomial space curves of arbitrary degree, J. Symb. Comput. 45, 844-856. MR2657668 
[17] C. Y. Han (2008), Nonexistence of rational rotation-minimizing frames on cubic curves, Comput. Aided Geom. Design 25, 298-304. MR.2408093 (2009a:65035)

[18] B. Jüttler (2001), Hermite interpolation by Pythagorean hodograph curves of degree seven, Math. Comp. 70, 1089-1111. MR.1826577(2002c:65027)

[19] B. Jüttler and C. Mäurer (1999), Cubic Pythagorean hodograph spline curves and applications to sweep surface modelling, Comput. Aided Design 31, 73-83.

[20] B. Jüttler and C. Mäurer (1999), Rational approximation of rotation minimizing frames using Pythagorean-hodograph cubics, J. Geom. Graphics 3, 141-159. MR.1748024 (2001c:53007)

[21] F. Klok (1986), Two moving coordinate frames for sweeping along a 3D trajectory, Comput. Aided Geom. Design 3, 217-229. MR871115 (88b:53001)

[22] E. Kreyszig (1959), Differential Geometry, University of Toronto Press. MR 0108795|(21:7507)

[23] J. Roe (1993), Elementary Geometry, Oxford University Press.

[24] Z. Sir and B. Jüttler (2007), $C^{2}$ Hermite interpolation by Pythagorean hodograph space curves, Math. Comp. 76, 1373-1391. MR2299779 (2008f:65035)

[25] W. Wang and B. Joe (1997), Robust computation of the rotation minimizing frame for sweep surface modelling, Comput. Aided Design 29, 379-391.

[26] W. Wang, B. Jüttler, D. Zheng, Y. Liu (2008), Computation of rotation minimizing frames, ACM Trans. Graphics 27, No. 1, Article 2, 1-18.

Department of Mechanical and Aerospace Engineering, University of California, Davis, CAlifornia 95616

E-mail address: farouki@ucdavis.edu

Dipartimento di Sistemi e Informatica, Università degli Studi di Firenze, Viale MorGAGNi 65, 50134 Firenze, ItALY

E-mail address: giannelli@dsi.unifi.it

Dipartimento di Matematica, Università di Roma "Tor Vergata," Via della Ricerca Scientifica, 00133 Roma, Italy

E-mail address: manni@mat.uniroma2.it

Dipartimento di Matematica “Ulisse Dini," Università degli Studi di Firenze, Viale Morgagni 67A, 50134 Firenze, Italy

E-mail address: alessandra.sestini@unifi.it 